Which indicators matter? Analyzing the Swiss business cycle using a large-scale mixed-frequency dynamic factor model

\author{
Alain Galli \\ SNB Working Papers \\ 8/2017
}




\section{Legal Issues}

\section{DISCLAIMER}

The views expressed in this paper are those of the author(s) and do not necessarily represent those of the Swiss National Bank. Working Papers describe research in progress. Their aim is to elicit comments and to further debate.

\section{COPYRIGHT@}

The Swiss National Bank (SNB) respects all third-party rights, in particular rights relating to works protected by copyright (information or data, wordings and depictions, to the extent that these are of an individual character).

SNB publications containing a reference to a copyright (C) Swiss National Bank/SNB, Zurich/year, or similar) may, under copyright law, only be used (reproduced, used via the internet, etc.) for non-commercial purposes and provided that the source is mentioned. Their use for commercial purposes is only permitted with the prior express consent of the SNB.

General information and data published without reference to a copyright may be used without mentioning the source. To the extent that the information and data clearly derive from outside sources, the users of such information and data are obliged to respect any existing copyrights and to obtain the right of use from the relevant outside source themselves.

\section{LIMITATION OF LIABILITY}

The SNB accepts no responsibility for any information it provides. Under no circumstances will it accept any liability for losses or damage which may result from the use of such information. This limitation of liability applies, in particular, to the topicality, accuracy, validity and availability of the information.

ISSN 1660-7716 (printed version)

ISSN 1660-7724 (online version)

(C) 2017 by Swiss National Bank, Börsenstrasse 15,

P.O. Box, $\mathrm{CH}-8022$ Zurich 


\title{
Which indicators matter? Analyzing the Swiss business cycle using a large-scale mixed-frequency dynamic factor model*
}

\author{
Alain Galli \\ Swiss National Bank and University of Bern ${ }^{\dagger}$
}

August 15, 2017

\begin{abstract}
For policy institutions such as central banks, it is important to have a timely and accurate measure of past and current economic activity and the business cycle situation. The most prominent example for such a measure is gross domestic product (GDP). However, GDP is only released at a quarterly frequency and with a substantial delay. Furthermore, it captures elements that are not directly linked to the business cycle and the underlying momentum of the economy. In this paper, I construct a new business cycle index for the Swiss economy, which uses state-of-the-art methods, is available at a monthly frequency and can be calculated in real-time, even when some indicators are not yet available for the most recent periods. The index is based on a large and broad set of monthly and quarterly indicators. As I show, for the case of Switzerland, it is important to base a business-cycle index on a broad set of indicators instead of only a small subset. This result contrasts with the results for other countries.
\end{abstract}

JEL classification: C32, C38, C53, C55, E32

Keywords: Business cycle index, dynamic factor model, mixed frequency, Switzerland

\footnotetext{
${ }^{*}$ I want to thank Marta Banbura, Gregor Bäurle, Christian Hepenstrick, Daniel Kaufmann, Matthias Lutz, Massimiliano Marcellino, Klaus Neusser, Alexander Perruchoud, Rolf Scheufele, an anonymous referee as well as the participants at the 2016 CIRET conference in Copenhagen and the SNB brownbag workshop for valuable comments. The views, opinions, findings, and conclusions or recommendations expressed in this paper are strictly those of the author. They do not necessarily reflect the views of the Swiss National Bank. The SNB takes no responsibility for any errors or omissions in, or for the correctness of, the information contained in this paper.

${ }^{\dagger}$ Swiss National Bank, P.O. Box, Börsenstrasse 15, 8022 Zürich. E-Mail: alain.galli@snb.ch.
} 


\section{Introduction}

For policy institutions such as central banks, it is important to have a timely and accurate measure of past and current economic activity and the business cycle situation. The most prominent example for such a measure is gross domestic product (GDP). However, GDP is only released at a quarterly frequency and with a substantial delay. In Switzerland, for instance, quarterly GDP is published approximately two months after the respective quarter has ended.

In addition to this timeliness issue, there is a second potential caveat regarding the use of GDP as the main business cycle measure: GDP also captures elements that are not directly linked to the business cycle and the underlying momentum of the economy. Examples of such elements are non-cyclical GDP components such as public spending and value added of the public sectors, or transitory components (idiosyncratic events, weather effects, etc.).

Finally, the specific way quarterly GDP is calculated usually implies that it is revised late, often quite substantially, e.g., by benchmark revisions, new annual GDP figures or changes in the underlying calculation methods. Such ex-post changes in a business-cycle measure are problematic from a policymaking perspective.

Therefore, to assess the current state of the economy (a) in a more timely manner and at a higher frequency, (b) in a more cycle-oriented manner and (c) in a way that makes them less prone to revisions, policymakers often use alternative measures of economic activity and conditions. Prominent examples of such business cycle measures are the Aruoba-Diebold-Scotti Business Conditions Index (see Aruoba, Diebold, and Scotti, 2009), the Federal Reserve Bank of Philadelphia State Coincident Indexes (see Crone and Clayton-Matthews, 2005) and the Conference Board Coincident Economic Index (see The Conference Board, 2001) for the US or the EuroCOIN index (see Altissimo, Bassanetti, Cristadoro, Forni, Lippi, Reichlin, and Veronese, 2001, Altissimo, Cristadoro, Forni, Lippi, and Veronese, 2010), the EuroMInd index (see Frale, Marcellino, Mazzi, and Prioetti, 2010) and the EuroSTING index (see Camacho and Perez-Quiros, 2010) for the euro area. For Switzerland, the KOF barometer (see Abberger, Graff, Siliverstovs, and Sturm, 2014) is currently the only (publicly available) broad-based business cycle measure. 
In this paper, I construct a new business cycle measure for the Swiss economy that uses state-of-the-art methods and (a) can be calculated in real-time, even when some indicators are not available yet for the most recent periods (ragged edge setup), (b) is available at a monthly frequency, (c) incorporates a very large number of economic indicators (largescale setup) and (d) includes both quarterly and monthly indicators (mixed-frequency setup). The two last points are necessary since the index uses a large and broad-based set of monthly and quarterly indicators.

From a detailed assessment of the resulting business cycle index, five findings emerge. First, an analysis of the relative importance of the indicators for the business cycle index suggests that the 100 most important indicators account for only $52 \%$ of the index. This is in contrast to the results for other countries. The obtained business cycle index is therefore driven by a large and broad based set of indicators and not only by a small subset of variables. This makes the index much more robust.

Second, the contributions of the different indicators to the index over time show that although there is a considerable degree of comovement across indicators, there can also be periods over which the indicator categories (e.g., industry sector, foreign trade, construction sector, labor market, etc.) and indicator types (e.g., surveys, hard data, financial indicators, foreign indicators) differ more substantially from each other.

Third, an analysis of the contribution of news within the real-time data flow sheds light on how the business cycle index takes into account new data that have been released since the last estimation. This suggests that financial variables are the main source of news to the model within the target month itself, surveys in the month after and hard data two months after.

Fourth, a more detailed investigation of the role of financial and foreign variables shows that, overall, an index based only on domestic non-financial indicators would be very similar to the index including all variables. However, focusing solely on the peaks and troughs, there are considerable differences. For instance, not including financial and foreign variables would miss the recession in $2002 / 3$ and point to a much stronger acceleration after the financial crisis in 2008 than was actually the case in terms of GDP developments. The reduced business cycle index also sees a somewhat more intense downturn after the exchange rate appreciation in 2011 and 2015 and a more sluggish recovery thereafter than 
the official GDP figures suggest.

Fifth, analyzing the sensitivity of the new business-cycle index to the real-time data flow reveals that the index becomes quite accurate already 30 days after the respective target month has ended. Accuracy gains are highest in the target month itself and the month after.

The remainder of this paper is organized as follows. Section 2 outlines the modeling approach and section 3 describes the data. Section 4 presents the resulting business cycle index and its key characteristics, both in terms of its performance as a measure of economic activity and with respect to the contributions of the various indicator groups. Finally, section 5 concludes.

\section{Modeling a business-cycle indicator}

This section gives an overview on the econometric approach on which the business-cycle indicator will be based.

\subsection{The dynamic factor model}

The most popular approach to constructing a business cycle measure that is based on a large set of indicators is the use of factor models. Different versions of factor models have been presented in the literature, e.g., in Stock and Watson (2002a,b, 2011), Bai (2003), Giannone, Reichlin, and Small (2008), Matheson (2011), Matheson (2012), Altissimo, Bassanetti, Cristadoro, Forni, Lippi, Reichlin, and Veronese (2001), or Forni, Hallin, Lippi, and Reichlin $(2000,2001)$. All of them have in common the fact that a set of indicators is explained by a small number of common factors that drive the variables included in the model. The approaches mainly differ in their scale (small versus large), the frequency of the included indicators (only monthly versus both monthly and quarterly), the specification (static versus dynamic), the econometric technique to extract the factors (principal components versus Kalman filter) and the way they handle missing observations due to mixed frequencies and ragged edges.

The business cycle indicator for the Swiss economy proposed in this paper is based on a large data set with indicators at both monthly and quarterly frequency. Therefore, 
a mixed-frequency large-scale version of the dynamic factor model is used. Following Mariano and Murasawa (2010), our monthly data vectors are defined as follows:

$$
y_{t}:=\left[\begin{array}{c}
G D P_{t} \\
x_{t}
\end{array}\right]=\left[\begin{array}{c}
G D P_{t} \\
x_{t}^{Q} \\
x_{t}^{M}
\end{array}\right], y_{t}^{*}:=\left[\begin{array}{c}
g d p_{t}^{*} \\
x_{t}^{*}
\end{array}\right]=\left[\begin{array}{c}
g d p_{t}^{*} \\
x_{t}^{Q^{*}} \\
x_{t}^{M}
\end{array}\right] .
$$

The observed, mixed-frequency data vector $y_{t}$ consists of quarterly GDP $\left(G D P_{t}\right)$ and a set of $n-1$ economic variables $\left(x_{t}\right)$, which contains both quarterly indicators $\left(x_{t}^{Q}\right)$ and monthly indicators $\left(x_{t}^{M}\right)$. The quarterly part of $y_{t}$ is observed only every third month. Latent values are replaced by zero. In the purely monthly equivalent of $y_{t}, y_{t}^{*}$, the monthly values for the quarterly variables $\left(g d p_{t}^{*}\right.$ and $\left.x_{t}^{Q^{*}}\right)$ are latent. Non-stationary indicators enter either in 3-month differences or growth rates in the case of monthly variables and in either quarter-on-quarter differences or growth rates in the case of quarterly variables.

The relationship between the variables, $y_{t}$, and the $r$ (unobserved) factors $f_{t}$ on a monthly basis is then defined as

$$
y_{t}^{*}=\Lambda f_{t}+u_{t}
$$

$\Lambda$ denotes the factor loadings and $u_{t}$ the idiosyncratic part of the data, which cannot be explained by the common component $\Lambda f_{t}$. The error-terms $u_{t}$ could potentially be autocorrelated, so that

$$
u_{t}=\Theta u_{t-1}+w_{t} \text { where } w_{t} \stackrel{i i d}{\sim} N\left(0, \Sigma_{w w}\right)
$$

Within the standard dynamic factor framework, the factors are assumed to follow a $\operatorname{VAR}(p)$-process:

$$
f_{t}=\underset{r \times r}{\Phi_{1}} f_{t-1}+\ldots+\underset{r \times r}{\Phi_{p}} f_{t-p}+v_{t}, \text { where } v_{t} \sim N\left(0, \Sigma_{v v}\right) .
$$

\subsection{Time aggregation linkages}

Given the mixed-frequency setup we are working with, we need to define time aggregation rules for the variables that are only observed at a quarterly frequency. For the quarterly 
stock variables in $y_{t}$, the aggregation is performed in level terms, so that the link between the quarterly levels $\left(x_{i, t}^{Q}\right.$, observed only every third month) and the unobserved monthly levels, $x_{i, t}^{Q^{*}}$, is given by

$$
x_{i, t}^{Q}=\left(\frac{1}{3}+\frac{1}{3} L+\frac{1}{3} L^{2}\right) x_{i, t}^{Q^{*}}=G(L) x_{i, t}^{Q^{*}} \quad \text { for } \mathrm{t}=3,6,9, \ldots
$$

This states that the quarterly level of an indicator corresponds to the simple average of its monthly levels within the respective quarter. ${ }^{1}$

For the quarterly flow variables in $y_{t}$, the aggregation is performed in terms of level changes. However, since the monthly flow variables enter the model via 3-month (and not month-on-month) changes, the aggregation rule turns out to be equal to (5) for the quarterly stock variables: the quarterly change of an indicator is given by the simple average of its 3 -month changes within the respective quarter. ${ }^{2}$

Written in terms of $y_{t}$ and $y_{t}^{*}$, we therefore obtain the following time aggregation relationship for time periods where the quarterly values are observed $(\mathrm{t}=3,6,9, \ldots)$ :

$$
y_{t}=(\underbrace{\left[\begin{array}{cc}
\frac{1}{3} I_{n^{Q}} & \mathbf{O} \\
\mathbf{O} & I_{n^{M}}
\end{array}\right]}_{G_{0}}+\underbrace{\left[\begin{array}{cc}
\frac{1}{3} I_{n} Q & \mathbf{O} \\
\mathbf{O} & \mathbf{O}
\end{array}\right]}_{G_{1}} L+\underbrace{\left[\begin{array}{cc}
\frac{1}{3} I_{n^{Q}} & \mathbf{O} \\
\mathbf{O} & \mathbf{O}
\end{array}\right]}_{G_{2}} L^{2}) y_{t}^{*} .
$$

For the remaining time periods $(t \neq 3,6,9, \ldots)$, we obtain:

$$
y_{t}=\underbrace{\left[\begin{array}{cc}
\mathbf{O}_{n^{Q}} & \mathbf{O} \\
\mathbf{O} & I_{n^{M}}
\end{array}\right]}_{G} y_{t}^{*} .
$$

Combining the dynamic factor model and the time aggregation equations, we obtain

\footnotetext{
${ }^{1}$ An alternative would be that the quarterly level equals the level at the last day of the respective quarter. However, in the context of business cycle analysis this would not be appropriate since such an aggregation would miss temporary shocks that have occurred during the quarter. Furthermore, quarterly surveys usually ask about quarterly averages and not end-of-quarter values.

${ }^{2}$ Note that in Mariano and Murasawa (2003) or Banbura, Giannone, Modugno, and Reichlin (2013), the monthly flow series are included as monthly (and not 3-month) changes. In this case, the aggregation rule for the quarterly flow variables would change to $G^{f}(L)=\frac{1}{3}+\frac{2}{3} L+\frac{3}{3} L^{2}+\frac{2}{3} L^{3}+\frac{1}{3} L^{4}$. However, as experiments showed, applying their aggregation rule leads to inferior forecasting results for Switzerland. Therefore, I apply the aggregation rule proposed by Angelini, Camba-Mendez, Giannone, Reichlin, and Rünstler (2011).
} 
the following relationship between the observed variables $y_{t}$, the monthly factors $f_{t}$ and the monthly errors $u_{t}$ :

$$
\begin{gathered}
y_{t}=\mathbf{G}_{t}(L) y_{t}^{*}=\mathbf{G}_{t}(L)\left(\Lambda f_{t}+u_{t}\right)=\mathbf{G}_{t}(L) \Lambda f_{t}+\mathbf{G}_{t}(L) u_{t}, \\
\text { where } \mathbf{G}_{t}(L)= \begin{cases}G_{0}+G_{1} L+G_{2} L^{2} & \text { for } \mathrm{t}=3,6,9, \ldots \\
G & \text { otherwise }\end{cases}
\end{gathered}
$$

\subsection{Choice of number of factors $(r)$ and lags $(p)$}

To select the number of factors $(r)$ that we want to include in the model, we use the Bayesian information criterion (BIC) for the measurement equation for GDP, i.e., $y_{t, g d p}=$ $\mathbf{G}_{t}(L) \lambda_{g d p} f_{t}+\mathbf{G}_{t}(L) u_{t, g d p}$ and choose the model with the lowest BIC. ${ }^{3}$ The BIC is given by

$$
B I C(r)=\ln \left(V\left[\mathbf{G}_{t}(L) \hat{u}_{t, g d p}\right]\right)+\frac{\ln (T)}{T} r
$$

An alternative is to select the number of factors according to an out-of-sample performance measure, such as the root mean squared forecast errors. This does not make a difference in our case. Using the data set outlined in the next section, both methods yield $r=4$.

Given the number of factors $r$, we choose the number of lags $p$ based on the $\operatorname{VAR}(p)$ of the factors that is associated with the lowest BIC. Following Luetkepohl (2005), pp. 140 and 150 , the $\operatorname{BIC}$ for a $\operatorname{VAR}(p)$ can be written as

$$
B I C(p)=\ln \left|\hat{\Sigma}_{v v}(p)\right|+\frac{\ln T}{T} p r^{2}
$$

where $\hat{\Sigma}_{v v}(p)=\frac{1}{T}\left(f_{t}-\hat{\Phi}_{1} f_{t-1} \ldots-\hat{\Phi}_{p} f_{t-p}\right)\left(f_{t}-\hat{\Phi}_{1} f_{t-1}-\ldots-\Phi_{p} f_{t-p}\right)^{\prime}$. Applying this criterion yields $p=1$.

For the business cycle index proposed in this paper, I do not explicitly model the autocorrelation in the idiosyncratic errors (i.e., $u_{t}$ is i.i.d.). Experimenting with different

\footnotetext{
${ }^{3}$ By selecting the number of factors based on the BIC, we follow Lopes and West (2004) and Song and Belin (2008) who showed that BIC is preferred to AIC when the number of factors is unknown. Furthermore, Lopes and West (2004) found that AIC tends to select too many factors.
} 
model specifications revealed that controlling for autocorrelated errors worsens both the in-sample and the out-of-sample performance of the model.

\subsection{Estimation of the parameters and the model}

Given the large scale and the high number of missing observations due to the mixedfrequency setup and the ragged edges, the model cannot be estimated by standard, gradient-based maximum likelihood approaches (e.g., as used by Mariano and Murasawa, 2010) in our case. Instead, we apply a 2-step estimation method along the lines of Giannone, Reichlin, and Small (2008) and Doz, Giannone, and Reichlin (2011). ${ }^{4}$ First, the parameters of the model are estimated by the following steps:

1. Perform a principal component analysis over a balanced data set containing only the monthly variables. ${ }^{5}$

2. Estimate $\Lambda$ by regressing all variables on the first $r$ principal components using the following regression setup:

- For quarterly variables: $x_{i, t}^{Q}=\Lambda_{i} G(L) f_{t}^{P C}+\tilde{u}_{i, t}$, where $\tilde{u}_{i, t}=G(L) u_{i, t}$.

- For monthly variables: $x_{i, t}^{M}=\Lambda_{i} f_{t}^{P C}+u_{i, t}$.

3. Estimate $\Sigma_{u u}$ using the residuals from the regressions above in the following way:

- For quarterly variables, $\tilde{u}_{t, i}=G(L) u_{i, t}$. Therefore, $V\left[\tilde{u}_{t}\right]=V\left[G(L) u_{t}\right]=$ $\frac{1}{9} V\left(u_{t}\right)+\frac{1}{9} V\left(u_{t-1}\right)+\frac{1}{9} V\left(u_{t-2}\right)=\frac{3}{9} V\left(u_{t}\right)$ and $V\left(u_{t}\right)=\frac{9}{3} V\left(\tilde{u}_{t}\right){ }^{6}$

- For monthly variables $V\left(u_{t}\right)=V\left(u_{t}\right)$.

4. Estimate $\Phi$ and $\Sigma_{v v}$ using a simple $\operatorname{VAR}(p)$ of the first $r$ principal components.

To make the model applicable to a mixed-frequency environment with ragged edges and missing values, we set it up in state-space form and compute it using the Kalman

\footnotetext{
${ }^{4}$ An alternative would be to use the EM algorithm as proposed in Doz, Giannone, and Reichlin (2012) and Banbura and Modugno (2014). However, experimenting with this procedure showed that it would result in very smooth factors and do worse than the two-step procedure in an out-of-sample nowcasting exercise.

${ }^{5}$ The minimum sample length for the balanced data set is set to 15 years.

${ }^{6}$ Note that the reason for not having to distinguish between quarterly flow and stock variables when estimating the variances is that our quarterly flow variables enter in 3-month growth changes. In the case of month-on-month growth rates, we would have that $V\left[\tilde{u}_{i, t}\right]=V\left[G^{f}(L) u_{i, t}\right]=\frac{1}{9} V\left(u_{i, t}\right)+\frac{4}{9} V\left(u_{i, t-1}\right)+$ $\frac{9}{9} V\left(u_{i, t-2}\right)+\frac{4}{9} V\left(u_{i, t-3}\right)+\frac{1}{9} V\left(u_{i, t-4}\right)=\frac{3}{9} V\left(u_{t}\right)$. Therefore, $V\left(u_{i, t}\right)=\frac{9}{3} V\left(\tilde{u}_{i, t}\right)$.
} 
smoother. This allows us to incorporate information that is only available at a quarterly frequency (e.g., quarterly indicators such as employment, consumer confidence or housing prices) and provides a timelier measure of current economic activity since we do not have to wait until all observations for the respective month are available. Furthermore, we are also able to include variables that are fairly new and therefore shorter than the balanced sample.

The measurement equation of the state-space system is given by the link between $y_{t}$ and $y_{t}^{*}$, expressed in terms of $f_{t}$ and $u_{t}$ as shown in equation (8):

$$
y_{t}=H_{t} \underbrace{\left[\begin{array}{c}
f_{t} \\
f_{t-1} \\
f_{t-2}
\end{array}\right]}_{\xi_{t}}+\underbrace{\left[\begin{array}{c}
G(L) u_{t}^{Q} \\
u_{t}^{M}
\end{array}\right]}_{\epsilon_{t}}
$$

where $H_{t}=\left\{\begin{array}{lll}{\left[\begin{array}{lll}G_{0} \Lambda & G_{1} \Lambda & G_{2} \Lambda\end{array}\right]} & \text { for } \mathrm{t}=3,6,9, \ldots \\ {\left[\begin{array}{ll}G \Lambda & \mathbf{O}_{n \times 2 r}\end{array}\right]} & \text { otherwise }\end{array}\right.$

and $\epsilon_{t} \sim N\left(\mathbf{0}_{n}, R\right)$, where $R=\left\{\begin{array}{l}{\left[\begin{array}{cc}\frac{3}{9} \Sigma_{w w}^{Q} & \mathbf{O}_{n^{Q} \times n^{M}} \\ \mathbf{O}_{n^{M} \times n^{Q}} & \Sigma_{w w}^{M}\end{array}\right] \quad \text { for } \mathrm{t}=3,6,9, \ldots} \\ {\left[\begin{array}{cc}\mathbf{O}_{n^{Q} \times n^{Q}} & \mathbf{O}_{n^{Q} \times n^{M}} \\ \mathbf{O}_{n^{M} \times n^{Q}} & \Sigma_{w w}^{M}\end{array}\right] \quad \text { otherwise }^{6}}\end{array}\right.$

For $t=3,6,9, \ldots$ and for all monthly variables, $y_{i, t}$ equals the actual observations of the respective indicator $i$. For $t \neq 3,6,9, \ldots, y_{i, t}$ is latent and set to zero when $i$ is a quarterly variable. The same applies to any other missing observations in the data set. More details on how the Kalman Filter is able to address missing observations can be found in Durbin and Koopman (2012). The transition equation of the state-space system represents the

\footnotetext{
${ }^{6}$ For numerical reasons ( $R$ must be invertible), we replace the upper left element by $c I_{n} Q$, where $c$ is fixed and a very small number, see Neusser (2011), p. 250.
} 
VAR of the factors:

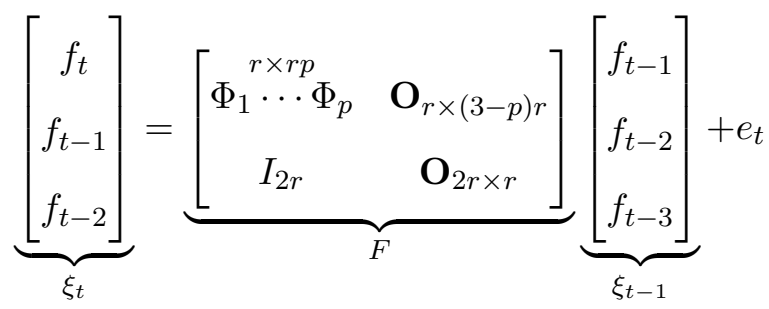

$$
\text { where } e_{t}=\left[\begin{array}{c}
v_{t} \\
\mathbf{0}_{2 r}
\end{array}\right] \sim N\left(\mathbf{0}_{3 r}, Q\right) \text {, where } Q=\left[\begin{array}{cc}
\Sigma_{v v} & \mathbf{O}_{r \times 2 r} \\
\mathbf{O}_{2 r \times r} & \mathbf{O}_{2 r \times 2 r}
\end{array}\right]
$$

Formed in state-space form, our mixed-frequency dynamic factor model now looks as follows:

$$
\begin{gathered}
y_{t}=H_{t} \xi_{t}+\epsilon_{t} \\
\xi_{t}=F \xi_{t-1}+e_{t} \\
\text { with }\left[\begin{array}{l}
e_{t} \\
\epsilon_{t}
\end{array}\right] \sim N\left[\begin{array}{ll}
Q & 0 \\
0 & R
\end{array}\right]
\end{gathered}
$$

Running the Kalman smoother on this model based on the parameters estimated previously yields reestimated monthly factors, which also take into account information obtained from quarterly variables, as well as monthly fitted values for all quarterly variables and forecasts for all variables. As a calibration measure that relates our four factors to the business cycle, GDP is used, implying that our business cycle index for the Swiss economy is given by the monthly fitted values for quarterly GDP and the related monthly forecasts.

\section{The data set}

In the context of constructing a business cycle index, the choice of the underlying data set is as important as the choice of modeling technique. In principle, a business cycle index could potentially be calculated based on a small data set of selected indicators, chosen by either statistical criteria or judgment. However, as shown in Galli, Hepenstrick, and Scheufele (2017), the obtained results from such small-scale models are not very promising 
for the case of Switzerland, especially in periods of large economic shocks. Therefore, the business cycle index proposed in this paper uses a large data set for the Swiss economy containing both monthly and quarterly indicators. The data set is very broad in two senses: It covers many different indicator categories (see below), and it also includes many different indicators per indicator category.

Two critiques are usually mentioned regarding the use of such a large data set. First, it could be argued that such a large-scale model contains too much noise and is not adequate for capturing the business-cycle correctly. It has been shown that a smallscale factor model can outperform larger factor models (see, e.g., Boivin and Ng, 2006), using disaggregate data can do worse than using aggregate data only (see, e.g., Banbura and Modugno, 2014), or that it may well be important to be broad in the sense of using many different data categories, but not necessarily in the sense of using more than one indicator per category (see, e.g. Alvarez, Camacho, and Perez-Quiros, 2016). Following these arguments of using a smaller model, however, always involves the application of a data selection process, either by judgment or by statistical criteria. This may not be straightforward in our case, since the variable of interest, i.e., the business cycle, is unobserved. ${ }^{7}$ Experimenting with such selection procedures shows that, in our case, not only would such a data reduction fail in improving the model, it would also have the effect that one single indicator has a much higher influence on the estimated business cycle index. This would make the index much more prone to indicator issues that an applied economist faces in practice, such as indicators with large publication lags, indicators that are temporarily or permanently discontinued, or indicators that are available very timely but that are comparatively volatile. In a large-scale model, in contrast, it needs a much broader-based movement among indicators to significantly influence the index. In addition, a large-scale model also has the benefit that it allows a detailed assessment of the whole space of available indicators within one framework.

The second critique that is sometimes mentioned is related to the use of quarterly variables. It could be argued that these are only of limited interest for a monthly business

\footnotetext{
${ }^{7} \mathrm{~A}$ possibility would be to use GDP as the target variable for the selection of relevant indicators. However, this could potentially yield a set of indicators that are particularly good at explaining quarterly volatility in GDP but not the business cycle. Examples would be indicators that cover public spending, the health sector or sectors that are less linked to the overall business cycle.
} 
cycle measure and that the focus of the data set should be on rapidly available monthly indicators. However, there is usually a trade-off between timeliness and reliability/relevance. While indicators that are released early (such as leading indicators or financial variables) provide very timely information about the respective period, the information content, reliability and relevance of such indicators may at the same time be limited. Since the focus of our business-cycle index shall not be just on timeliness but also on accuracy, it is important to include all available information and not only the best in terms of rapid availability. This especially applies to the case of Switzerland, where several important indicators are only available at the quarterly and not the monthly frequency. Prominent examples are industrial production, industrial orders, employment data, consumer confidence or housing prices. Fortunately, there are econometric methods that help to reduce this trade-off between timeliness and accuracy. By always using all information available for the period of interest, our method allows us to obtain (a) the best-possible estimate of current economic activity on the basis of all indicators that have already been released and (b) an ex-post assessment of past economic conditions on the basis of the full information set.

Table 1 provides an overview of the data set. In total, it consists of 620 variables (362 monthly, 285 quarterly), covering 17 indicator categories. All data are used in calendar-, seasonally- and outlier-adjusted terms. ${ }^{8}$ The data set includes approximately 80 foreign indicators that capture foreign (especially euro-area and German) economic activity, which is important for a small-open economy such as Switzerland. The data set begins in 1975, but as shown in Figure B.5 in the Appendix many Swiss indicators start only in 1990 or even later and are therefore rather short compared to other countries. Figure B.5 also illustrates the ragged edge issue, with indicators that have very timely availability and others that have a publication lag of 2-3 months or even more.

In terms of indicator types, the data set consists of four different types: (I) hard data (turnover, orders, employment, unemployment, prices, etc.), (II) soft data (surveys), (III) foreign indicators and (IV) financial indicators. As shown in the left panel of Figure 1, surveys have the largest share of the data set (43\%), followed by hard data (36\%). The

\footnotetext{
${ }^{8}$ Indicators not available on a seasonally adjusted basis are calendar-, seasonally- and outlier-adjusted using the X-13ARIMA-SEATS procedure.
} 
Table 1: Large-scale data set for the Swiss economy

\begin{tabular}{|c|c|c|c|c|c|}
\hline \multirow[t]{2}{*}{ Category } & \multicolumn{2}{|c|}{ Soft data } & \multicolumn{2}{|c|}{ Hard data } & \multirow[t]{2}{*}{ Prominent examples } \\
\hline & $\mathrm{m}$ & $q$ & $\mathrm{~m}$ & $q$ & \\
\hline GDP & & & & 27 & $\begin{array}{l}\text { GDP, demand components, value added of } \\
\text { sectors. }\end{array}$ \\
\hline Labor market & 1 & 4 & 39 & 57 & $\begin{array}{l}\text { OASI statistics, unemployment statistic, } \\
\text { jobs statistic, employment statistics, sur- } \\
\text { veys, hours worked, wage index. }\end{array}$ \\
\hline Consumption & & 13 & 10 & & $\begin{array}{l}\text { Overnight stays of domestic visitors, re- } \\
\text { tail sales, import of fuel, electricity con- } \\
\text { sumption, new car registrations, consumer } \\
\text { sentiment. }\end{array}$ \\
\hline Investment & & & 3 & 6 & $\begin{array}{l}\text { SwissMEM survey, imports of investment } \\
\text { goods, industrial production of investment } \\
\text { goods. }\end{array}$ \\
\hline Foreign trade & & & 19 & 2 & $\begin{array}{l}\text { Trade statistics, overnight stays of foreign } \\
\text { visitors. }\end{array}$ \\
\hline Foreign activity & 24 & 13 & 39 & 8 & $\begin{array}{l}\text { Several indicators covering Germany, euro } \\
\text { area, United States, Japan, emerging Asia } \\
\text { and the CESifo world economic survey. }\end{array}$ \\
\hline Financial markets & & & 66 & & $\begin{array}{l}\text { Stock market indexes, exchange rates, } \\
\text { commodity price indexes, monetary aggre- } \\
\text { gates, monetary conditions, interest rates, } \\
\text { spreads. }\end{array}$ \\
\hline Prices & & & 11 & 3 & $\begin{array}{l}\text { Consumer prices, real estate prices, im- } \\
\text { port prices, production prices, construc- } \\
\text { tion prices. }\end{array}$ \\
\hline Construction sector & 15 & 3 & 7 & 24 & $\begin{array}{l}\text { Surveys, production, order books, cement } \\
\text { deliveries. }\end{array}$ \\
\hline Retail trade sector & 6 & 5 & & & Surveys. \\
\hline Wholesale trade sector & & 14 & & & Surveys. \\
\hline Accommodation sector & & 18 & 1 & & Overnight stays, surveys. \\
\hline Manufacturing sector & 51 & 39 & 4 & 4 & $\begin{array}{l}\text { Industrial production, surveys, PMI, elec- } \\
\text { tricity production, number of working } \\
\text { days. }\end{array}$ \\
\hline Project engineering sector & 8 & 7 & & & Surveys. \\
\hline Banking sector & 20 & 22 & 14 & & $\begin{array}{l}\text { Credit statistics, surveys, balance sheet } \\
\text { statistics, illiquidity index. }\end{array}$ \\
\hline Insurance sector & 16 & 9 & & & Surveys. \\
\hline Other services sector & & 6 & & & Surveys. \\
\hline Other indicators & 7 & 14 & 4 & & $\begin{array}{l}\text { Electricity production, cashless payment } \\
\text { transactions, bankruptcy statistics, sur- } \\
\text { veys. }\end{array}$ \\
\hline
\end{tabular}


Figure 1: Data set shares by indicator types and indicator frequencies
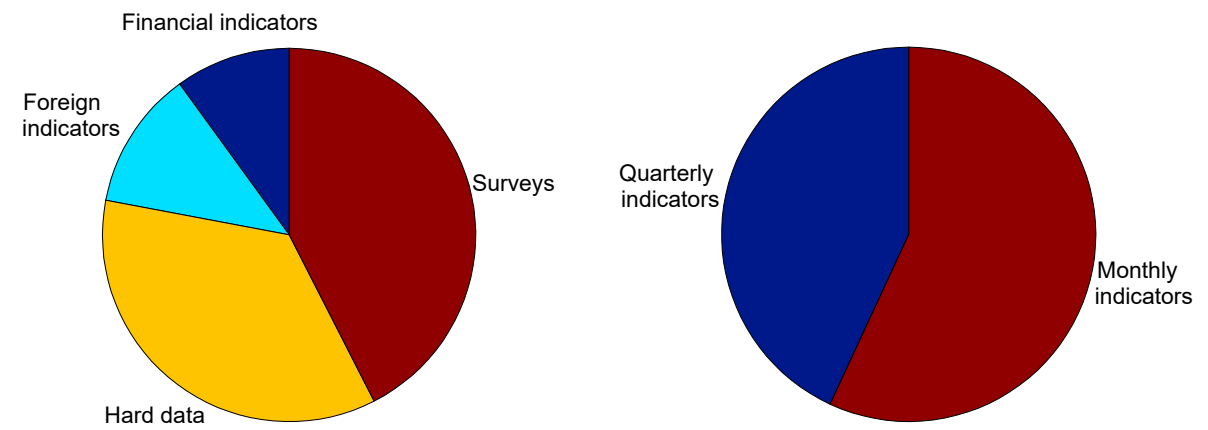

Note: Shown are the shares of the respective indicator type (left panel) and indicator frequency (right panel) in the entire data set.

Table 2: Stylized characteristics of different indicator types

\begin{tabular}{l|c|c|c|}
\hline Indicator type & Timeliness & Revision likelihood & Reliability and Relevance \\
\hline Hard data & Low to medium & Medium to high & Very high \\
Soft data & Medium & None to medium & High \\
Foreign indicators & Medium to high & None to high & Medium to high \\
Financial indicators & High & None & Medium \\
\hline
\end{tabular}

remainder consists of foreign and financial variables.

The stylized characteristics of these different indicator types in terms of timeliness, revision likelihood and reliability and relevance for the Swiss real economy are shown in Table 2. As explained earlier, there usually is a trade-off between timeliness and reliability/relevance. Hard data are the most reliable and relevant indicators but are often published with a substantial delay and subject to revisions. Soft indicators, in contrast, are released earlier but consist of surveys and thus do not provide a direct measure of outcomes. This lowers their reliability and relevance to some extent. Foreign indicators are usually released earlier than their Swiss equivalents; however, their relevance depends heavily on the current transmission of foreign developments on the Swiss economy. Finally, financial indicators have the advantage that they are available very early (usually even on a daily basis) and not subject to revisions. Their relevance for the real economy, however, can sometimes be limited. 


\section{Analyzing the Swiss business cycle}

This section presents the resulting business cycle index and its key characteristics, both in terms of its performance as a measure of economic activity and with respect to the contributions of the various indicator groups. It also analyzes the sensitivity of the new business-cycle index to the real-time data flow.

\subsection{The Swiss business cycle}

Figure 2 presents our estimate for the business-cycle for the Swiss economy based on the modeling approach described in section 2 and the data set outlined in section 3, together with official quarter-on-quarter GDP growth and the recession dates from Siliverstovs (2013). ${ }^{9}$ It can be seen that the Swiss economy has gone through several ups and downs during the last 25 years. ${ }^{10}$

In the first half of the 1990s, the Swiss economy was hit by the end of the Swiss housing boom, a global recession and a restrictive monetary stance. This led to an extended phase in which GDP growth was very weak, including two recessionary phases in 1990/91 and 1992/93; the business cycle index (BCI) remained negative throughout this period. Around 1993/94, it seemed that the economy had finally started to recover, but this acceleration was only temporary. It was only in 1997, when the economy finally started to recover, reaching robust expansion momentum between mid-1997 and mid-1998. After a slight, temporary slow down in the following year, the pace of expansion accelerated again, reaching a record high level in mid-2000.

This trend was stopped with the collapse of the dot-com-bubble and the subsequent slowdown of the global economy. Swiss GDP growth slowed rapidly in 2001, and the Swiss economy entered another recessionary phase that almost qualified as a double-dip recession. In GDP terms, the formal recession occurred in 2002/3, but the entire period from the beginning of 2001 to mid-2003 saw basically no growth. During this phase, the business cycle index was also mostly in negative territory.

\footnotetext{
${ }^{9}$ Note that the recession dates from Siliverstovs (2013) were based on quarterly GDP figures released in September 2012, which did not yet include the ESA2010 benchmark GDP revision implemented in 2014.

${ }^{10}$ Note that there is a potential structural break in the GDP series since the ESA2010 benchmark GDP revision was only implemented back to 1995. Since the business cycle index parameters are based on a balanced sample that only includes the revised part of the GDP series, the BCI values for the pre-1995 period should be treated with caution.
} 
Figure 2: Monthly business cycle index for the Swiss economy

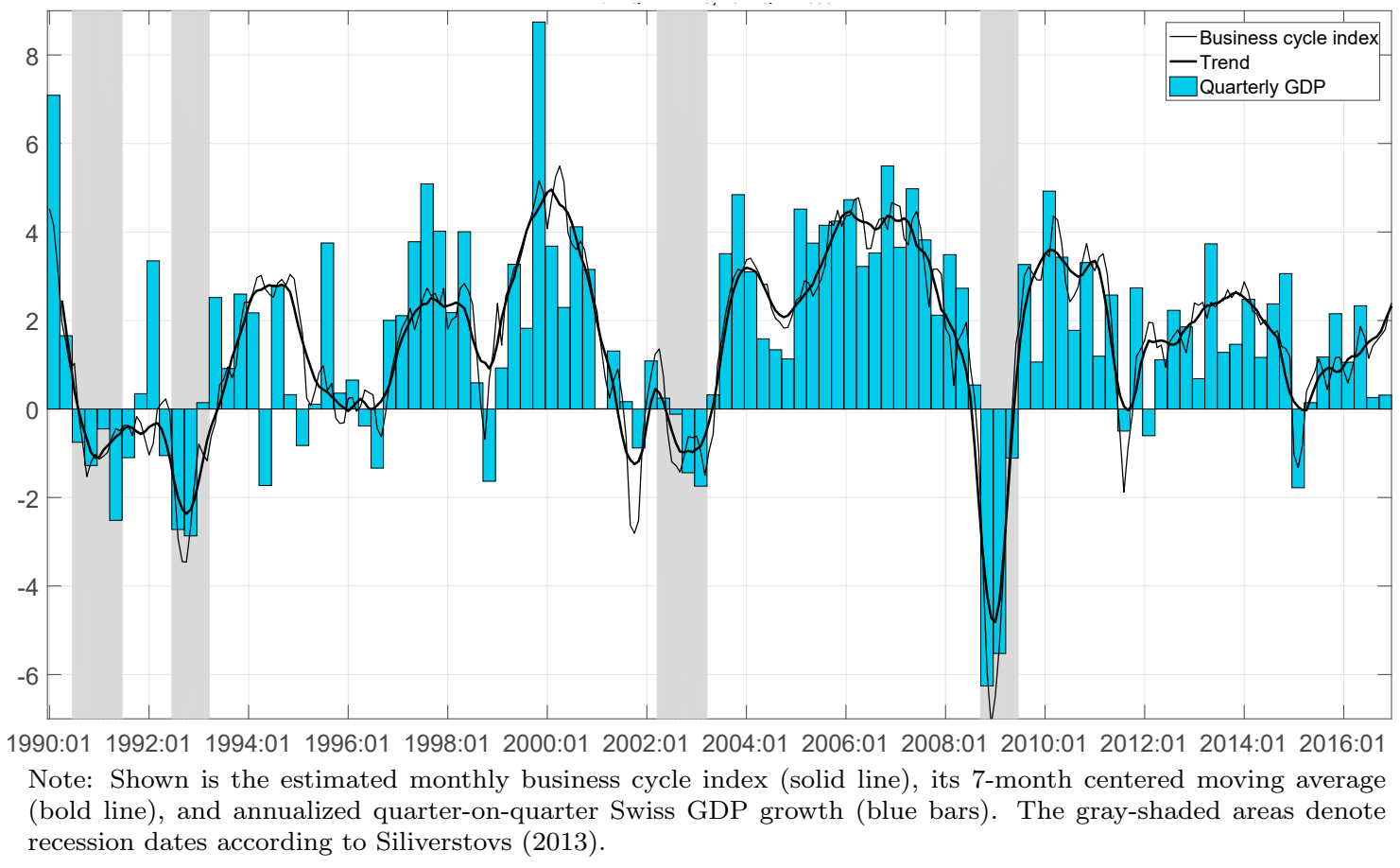

Starting in the second half of 2003, the Swiss economy entered an extended boom period. The economic momentum was particularly strong between 2005 and 2007. The boom was probably driven by two main developments. First, the Swiss economy was starting to benefit from bilateral agreements with the European Union, including a rapid increase in immigration, which boosted domestic demand. Second, this was a period with particularly strong global trade dynamics, especially due to the increasing role of China in world trade and the rapid expansion of global value chains. A third factor may have been the supportive effect of the extended phase in which the Swiss franc tended to weaken. The business cycle index tracked this phase very well, suggesting that the boom was very broad-based across different parts of the Swiss economy.

This phase came to an end in 2007/08, when economic momentum started to decline with the onset of the financial crisis, finally causing the economy to enter into recession between the end of 2008 and mid-2009. In parallel to GDP, the business cycle index contracted at a record pace in Q4 2008 and Q1 2009. The recession was sharp but also fairly short, and the economy began to recover during the second half of 2009. However, the recovery was neither particularly strong nor did it last very long. During 2010 and 2011, the Swiss economy was hit by the strong appreciation of the Swiss franc, leading to 
a slowdown in economic momentum. At the same time, the global economy slowed down, and the euro area, Switzerland's main trading partner, entered another recession. Swiss GDP was negative twice (in Q3 2011 and Q1 2012), while the business cycle index points to only three months of contraction (in Q3 2011). The appreciation was stopped by the Swiss National Bank's introduction of the minimum exchange rate against the euro on September 6, 2011. The business cycle index suggests that the floor led to a recovery in economic conditions, though the momentum in the following three years, during which the floor was in place, never reached the levels seen before and immediately after the financial crisis.

The discontinuation of the minimum exchange rate on January 15, 2015 led to another exchange rate shock. This caused another quarter of negative GDP growth and a three-month contraction of the business cycle index in Q1 2015. However, the Swiss economy did not enter a technical recession. In the subsequent quarters, the economy recovered steadily but gradually from the exchange rate shock.

Looking at the entire 1990-2016 period, the business cycle index follows the general tendency of GDP quite closely, with a correlation coefficient of 0.81 . Over the more recent past, 2000-2016, the relationship is even closer, with a correlation coefficient of 0.89 . However, there are also periods where the index deviates more persistently from GDP growth. For instance, in the years 1994 and 2013 the BCI was much more optimistic on economic conditions than GDP growth indicated. In contrast, the BCI was more pessimistic than GDP growth in 1997, indicating lower momentum, and in 2001, showing a stronger contraction in economic conditions. Such discrepancies between the business cycle index and GDP growth (both temporary or more persistent ones) can be driven by developments in GDP components that are not or only to a limited extent linked to the overall business cycle (such as public spending, value-added in the public sectors, production in the pharmaceutical sector, or merchanting) or by transitory GDP components (idiosyncratic events affecting certain sectors, such as weather effects, a shutdown of power plants or very sector-specific shocks). 


\subsection{Relative importance of indicators}

To assess the relative importance of the indicators for the business cycle index, the underlying factors (i.e., the first four states obtained from the Kalman smoother) are decomposed into the weighted sum of available information, i.e., the weighted sum of the indicators,

$$
f_{t \mid T}=\sum_{k=0}^{T} w_{k}(t, T) y_{k}
$$

where $w_{k}(t, T)$ denotes the weights attributed to the indicators of period $k$ for the factor estimates in $t$ based on all information available in $T$. These weights are calculated using the algorithm of Koopman and Harvey (2003), briefly summarized as follows:

1. In a first step, we calculate the weights for the filtered states from the Kalman filter. The weight of the indicators of period $k$ for the filtered states in $t$ based on all information available in $T$ is given by

$$
W_{k}^{f}(t, T)=B_{t, k} K_{k},
$$

where $K_{k}$ is the Kalman gain, which can be directly obtained from the Kalman filter (see Appendix A). $B_{t, k}=I$ for $k=t-1$ and $B_{t, k}=B_{t, k+1} F-w_{k+1}^{f}(t, T) H_{k}$ for $k<t-1$.

2. In a second step, we use the weights for the filtered states to calculate our object of interest, the weights for the smoothed states. The weight of the indicators of period $k$ for the smoothed states in $t$ based on all information available in $T$ is given by

$$
W_{k}(t, T)= \begin{cases}\left(I-P_{t \mid t-1} N_{t-1}\right) W_{k}^{f}(t, T) & \text { for } k<t \\ B_{t, k}^{*} C_{k} & \text { for } k \geq t\end{cases}
$$

with $C_{k}=H_{t}\left(S_{k}^{-1}+K_{k}^{\prime} N_{k} K_{k}\right)-F N_{k} K_{k}, B_{t, k}^{*}=P_{t \mid t-1}$ for $k=t$ and $B_{t, k+1}^{*}=B_{t, k}^{*} L_{k}^{\prime}$ for $k>t-1$. The terms $N_{k}=H_{k+1}^{\prime} S_{k+1}^{-1} H_{k+1}+L_{k+1}^{\prime} N_{k+1} L_{k+1}\left(\right.$ with $N_{T}=0$ ) and $L_{k}=F-K_{k} H_{t}$ depend on the Kalman gain. Together with the covariance matrix of the forecasted states, $P_{t \mid t-1}$, and the covariance of the Kalman filter forecast for $y_{k}$, $S_{k}$, this Kalman gain can be obtained directly from the Kalman filter (see Appendix 
Figure 3: Weights of indicators to factors
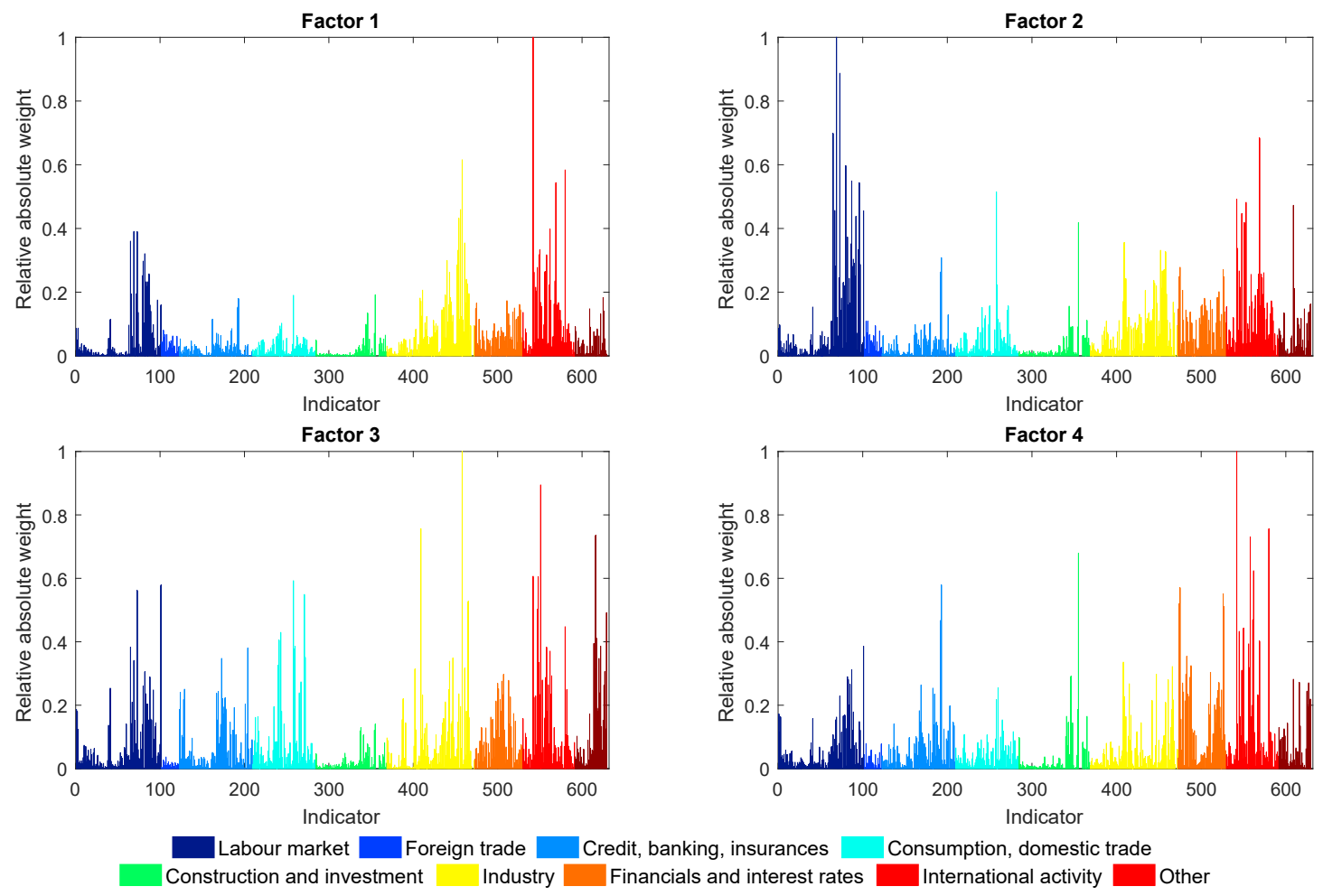

Note: Shown are the absolute factor weights of the indicators sorted by indicator group and relative to the most important indicator's weight for the respective factor.

A).

3. The indicator weights for our four BCI factors, $w_{k}(t, T)$, are finally obtained from the first four rows of $W_{k}(t, T) .{ }^{11}$

For each of the four factors, Figure 3 plots these indicator weights relative to the most important indicator for the respective factor. It is clearly visible that the relative importance of the indicators varies substantially across factors and across indicators.

Linear combinations of the indicator weights and the respective factor loadings for GDP yields each indicator's weight for the business cycle index. ${ }^{12}$ These BCI weights are shown in the left panel of Figure 4 relative to the most important indicator's weight. Ordering the indicators by their respective importance (in descending order) reveals that the relative importance decreases only very gradually. Even the 50th indicator has a weight

\footnotetext{
${ }^{11}$ Following Banbura and Ruenstler (2011), weights related to missing data are set to zero.

${ }^{12}$ Note that this procedure ignores potential correlation across factors, which could influence the indicators' weights for the business cycle index. One solution would be to include the BCI, i.e., the fitted value for GDP, in the state vector. However, in our case the correlations between the factors are comparatively low, so that influence of the weights should be limited.
} 
Figure 4: Weights of indicators to business cycle index
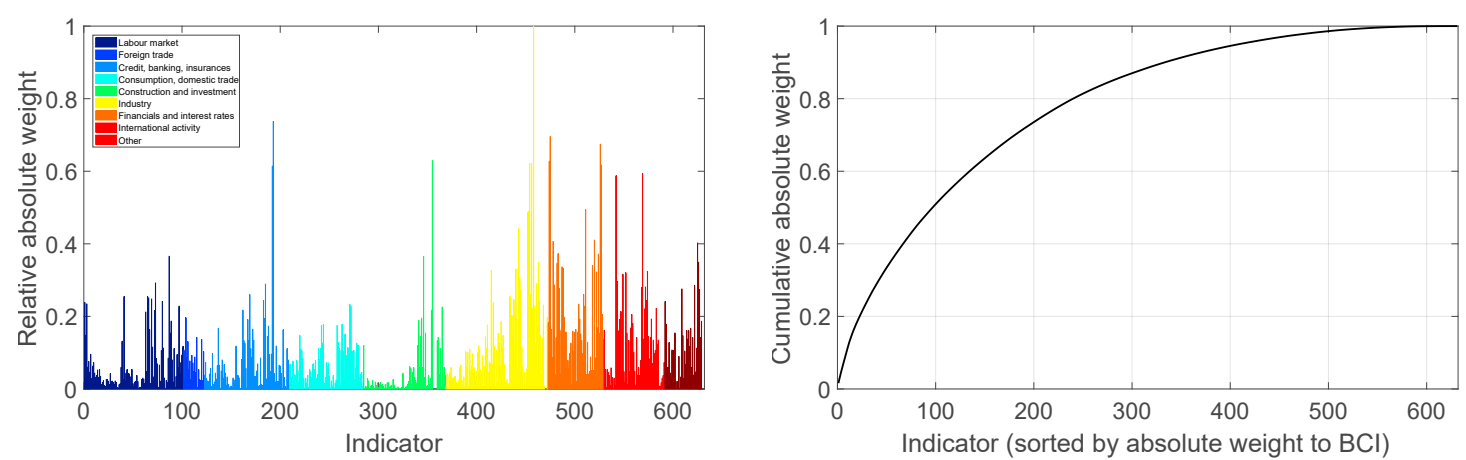

Note: The left panel shows the absolute BCI weight of the indicators sorted by indicator group and relative to most important indicator's weight. The right panel shows the cumulative absolute weight of the indicators in descending order according to their absolute BCI weight.

of $27 \%$ of the most important indicator's weight. In terms of the cumulative distribution of the ordered weights, shown in the right panel of Figure 4, the 25 most important indicators account for only $22 \%$ of the sum of total weights, the 100 most important indicators for only $52 \%$. This is in contrast to Banbura and Ruenstler (2011) who showed that for the euro area only a small set of indicators is of high importance. The obtained business cycle index for Switzerland is therefore driven by a large and broad-based set of indicators and not only by a small subset of variables. This makes the index much more robust.

Figure 5 shows the absolute weights of the 30 most important indicators, relative to the absolute weight of the most important indicator, which is the KOF industry survey on expected employment. ${ }^{13}$ At first glance, it may come as a surprise that Swiss stock market indexes (FINSPI, FINSMI), together with other financial indicators such as interest rates and exchange rates, are among the 30 most important indicators. This can be potentially explained by three channels. First, stock market performance is to a considerable extent linked to economic activity and conditions in Switzerland and abroad as well as to foreign stock market developments, especially in times of large economic shocks. Its weight for the first factor (which can be loosely interpreted as reflecting overall economic developments) is considerable. Second, when abstracting from foreign stock market developments, the Swiss stock market and the other financial indicators contain information on Swiss-specific developments and shocks. These seem to be captured by the model through the fourth factor, for which the weights of these indicators are also high. Finally, a third reason is

\footnotetext{
${ }^{13}$ The legend for the acronyms of the 30 most important indicators can be found in Table B.1 in the Appendix.
} 
Figure 5: 30 most important indicators in terms of their weight for the business cycle index

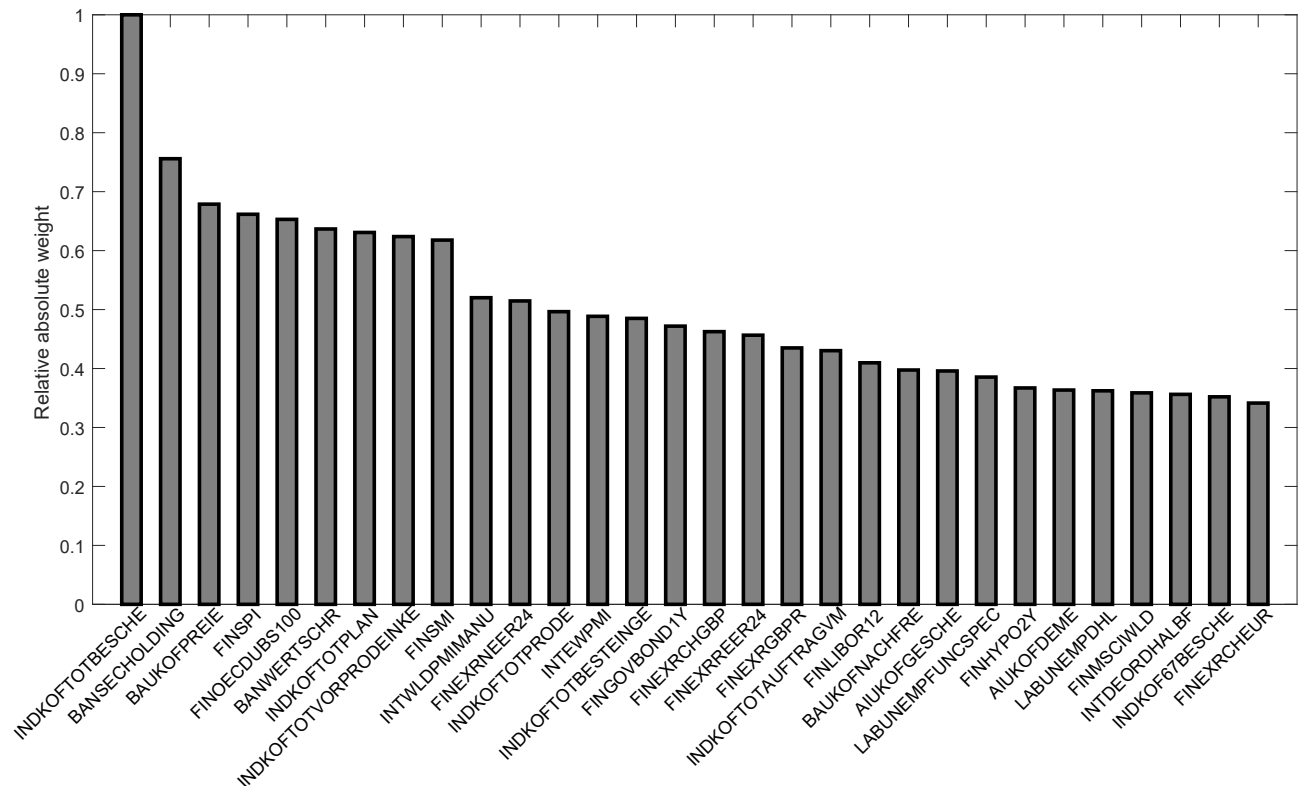

Note: Shown are the absolute weights of the 30 most important indicators relative to the most important indicator's weight. The legend for the acronyms of the 30 most important indicators can be found in Table B.1 in the Appendix.

that this fourth factor, in addition to its contemporaneous importance for the business cycle index, seems to also have some predictive power for the next period's first factor. This could reflect a confidence channel through which large changes in (Swiss-specific) financial conditions - with a slight delay - affect the general business cycle.

By dissecting the BCI-weights of these most important indicators into their weights for the four underlying BCI factors (scaled by the respective factor loading for the BCI), shown in Figure 6, we can characterize the 30 most important indicators by three main groups:

(I) Indicators that mainly affect the first factor (e.g., unemployment indicators and domestic surveys) are to a large extent directly related to the general business cycle

(II) Indicators with a positive effect on the first but a negative effect on the fourth factor (e.g., export-weighted PMI, world manufacturing PMI) mainly mirror the influence of foreign developments on the Swiss economy. If our business cycle index were to only incorporate the first factor, the impact of these foreign variables would to some extent be overstated in periods when more domestic shocks hit the Swiss economy (e.g., exchange rate appreciations or domestic stock market crashes). 
Figure 6: Decomposition of BCI weights of 30 most important indicators by factor

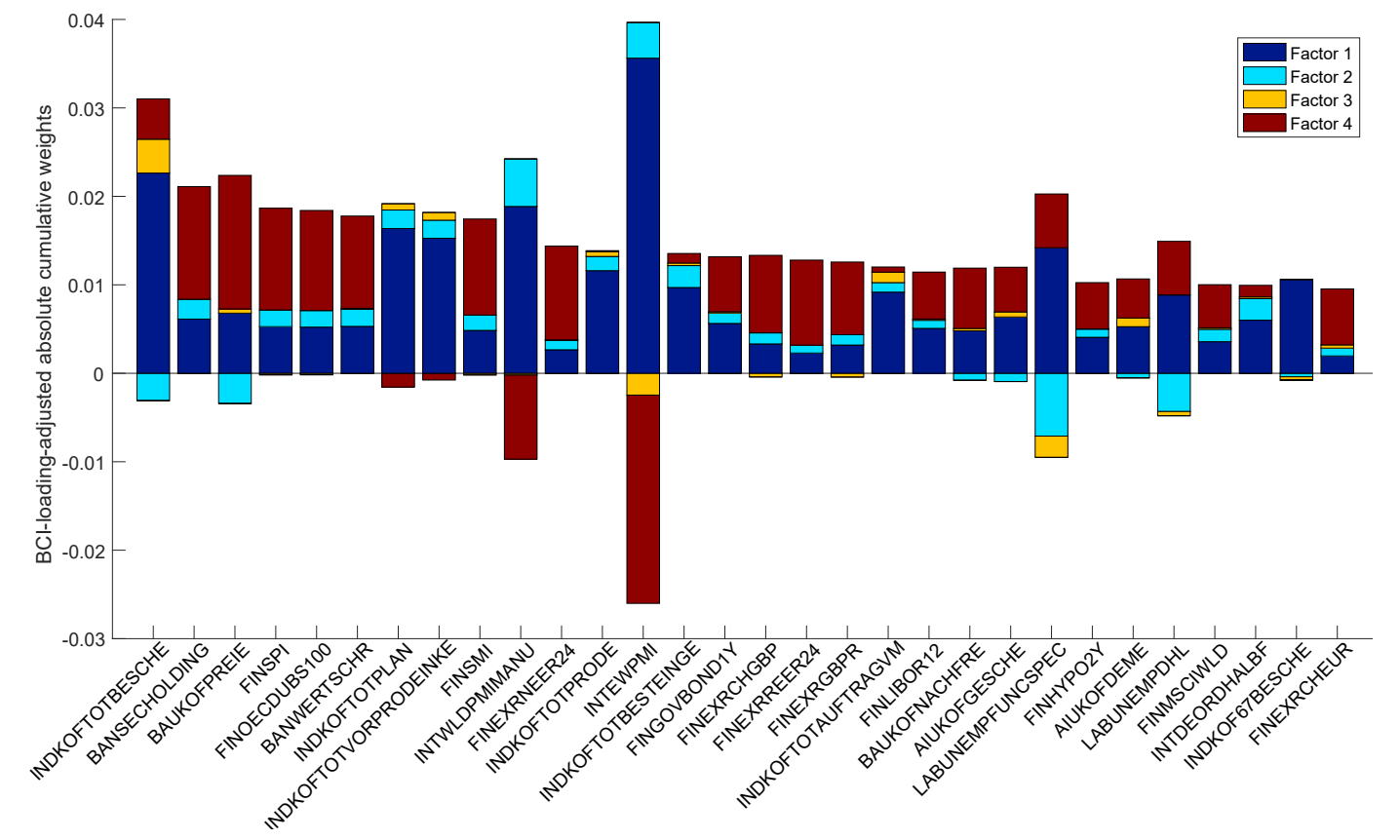

Note: The legend for the acronyms of the 30 most important indicators can be found in Table B.1 in the Appendix.

(III) Indicators with large weights for the fourth factor (Swiss stock market indexes, exchange rates, interest rates) are good at capturing Swiss-specific developments and shocks, especially domestic financial conditions.

\subsection{Business cycle contributions of indicator groups over time}

In a next step, I assess the contributions of the different indicator groups to the business cycle index over time. For each indicator $i$, its contribution to the four BCI factors can be calculated by $\sum_{k=0}^{t-1} w_{k, i}(t, T) y_{i, t}$, i.e., multiplying its period $t$ factor weights by the respective indicator observations.

\section{Contributions by indicator category}

Figure 7 shows the indicator contributions over time, aggregated by indicator category. The contributions help to distinguish different characteristics of the Swiss economy and the Swiss business cycle over the last 20 years. At first sight, there is a significant amount of comovement across the different indicator categories. Looking at the contributions in more detail, however, reveals some interesting additional aspects (see also Figure B.2 in the Appendix). 
Figure 7: Contributions to business cycle index by indicator categories

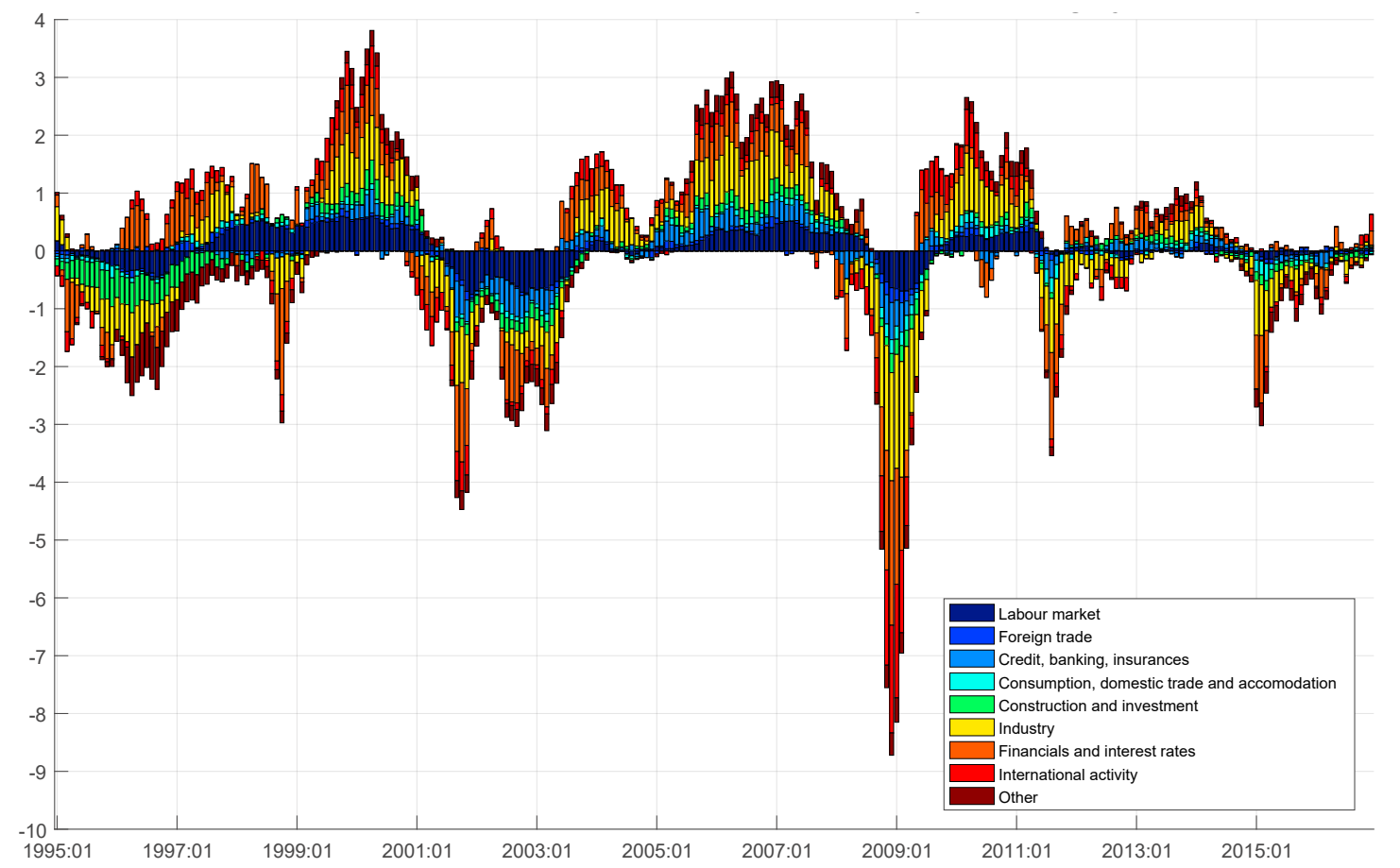

Note: Shown are the BCI contributions from the different indicator categories in deviations from mean.

Looking at the labor market indicators, their contributions turn out to be comparatively smooth over time and slightly lagging behind economic activity, which is a typical feature of the labor market found in applied economic research. Furthermore, they also reflect the severe deterioration in the labor market in 1995-1997, 2002/3 and 2009, which were characterized by increasing unemployment and falling employment. The effect of the exchange rate appreciation in 2011 and 2015, in contrast, was somewhat different. According to the labor market contributions to the business cycle index, these shocks had a less severe immediate labor market impact, but the effect was more persistent; unemployment increased at a much slower pace but more steadily than during the previous downturns and employment growth was somewhat lower but hardly ever negative.

The contributions also allow an analysis of how different economic downturns were reflected in the different indicator categories. For instance, the contribution from the industry indicators, the most cyclical category, was particularly negative during the financial crisis in $2008 / 2009$. The impact of the crisis in $2002 / 3$ and the exchange rate appreciations 2011 and 2015 were negative, too, but to a lesser extent than during the financial crisis. The contributions from domestic trade and accommodation indicators, in contrast, were 
as negatively hit by the exchange rate appreciations as by the financial crisis. Furthermore, while the recovery from the exchange rate shock was faster in 2011, supported by the introduction of the minimum EURCHF exchange rate by the Swiss National Bank, the weakness was more permanent in 2015/16. One reason for the stronger impact of the exchange rate appreciation on these indicator categories compared to industry-related indicators could be that domestic trade and accommodation are more exposed to the euro area, against whose currency the Swiss franc has appreciated the most over the preceding five years. Moving to the credit, banking and insurance category, the picture is different again: in terms of negative contribution impact, the dot-com bubble in 2002/ had as negative of an impact as the financial crisis. The exchange rate shocks in 2011 and 2015 also had a negative impact (also in combination with the current low interest environment), but to a lesser extent than in the $2002 / 3$ and 2009 crises.

What the contributions also illustrate is the permanent weakness in the construction sector in the 1990s and the high monthly volatility in foreign trade developments. Furthermore, they also show that the financial and foreign indicator categories tend to be less directly linked to the Swiss business cycle than domestic non-financial categories, but that they are also of high importance. When abstracting from the comparatively high monthly volatility, the contributions from financial variables not only nicely illustrate the weakness in financial conditions between 2000 and 2003 after the collapse of the dot-com bubble but also the large shocks in 2008 (Lehman Brothers), 2011 (large appreciation) and 2015 (end of minimum exchange rate). Foreign variables show the gradual deterioration of foreign business conditions during the financial crisis and the following strong recovery in 2009, reaching a level that was even higher than before the crisis. Only one year later, however, foreign business conditions deteriorated substantially again, driven by the European sovereign debt crisis. Although they recovered to some extent in 2013, they never reached pre-financial crisis levels again until very recently.

\section{Contributions by indicator type}

The indicator contributions to the business cycle over time can also be grouped by indicator type, as shown by the heat map in Figure 8. Several findings emerge. First, the contribution from surveys is more persistent, i.e., less volatile, than the one from hard 
Figure 8: Contributions to business cycle index by indicator types

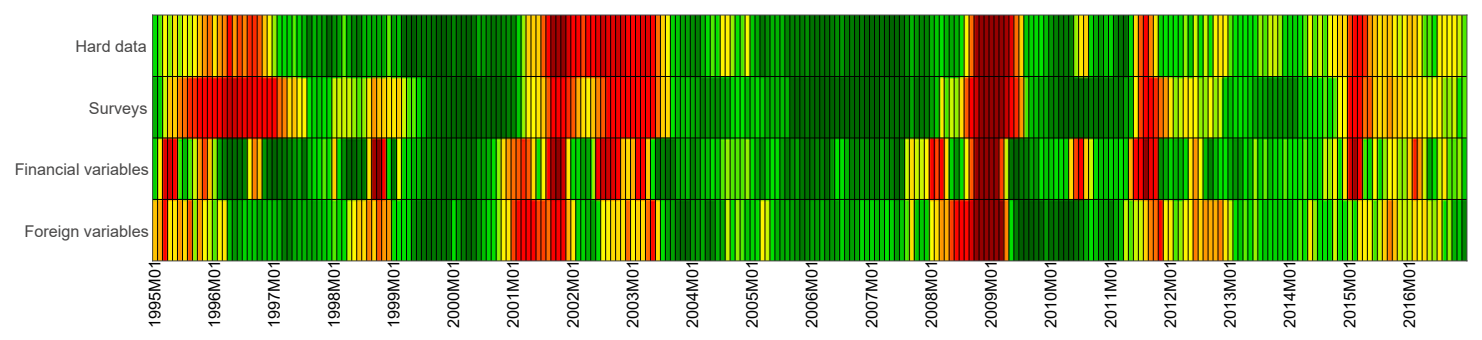

Note: This heat map shows the standardized BCI contributions from the different indicator types. The indicated momentum is coded by colors, which range from dark red (very strong contraction of economic activity) to dark green (very strong expansion). Yellow colors indicate average momentum.

data (see also Figures B.3 and B.4 in the Appendix). This mainly reflects the fact that hard data are usually more affected by transitory events such as weather effects, calendar effects and other idiosyncratic variations. Second, surveys and hard data generally follow the same tendencies, which suggests that surveys are a good overall proxy for real activity. Third, there can be periods where surveys deviate more substantially from hard data. For instance, surveys pointed to much stronger momentum during 2013 than hard indicators and to a more robust recovery in 2010. Conversely, they indicated much less momentum before the end of the dot-com bubble and a slower recovery from the exchange rate appreciation in 2011.

Fourth, the comparison between the contribution from foreign indicators on the one hand, and domestic hard and survey data on the other, illustrates nicely which foreign economic activity experienced stronger downturns (2001, 2008/9, 2013) and weaker downturns $(1995-7,2003,2011,2015)$ than the Swiss economy.

Fifth, the contribution from financial variables reveals that all domestic and foreign crises were also visible in financial indicators, either by (decreasing) interest rates, (drops in) stock market indexes, an (appreciating) Swiss franc or a combination of them.

\subsection{The role of news: which indicators matter?}

Each time the business cycle index is recalculated, it takes into account new data that have been released since the last estimation. The resulting BCI revision is, apart from potential revisions of past data, determined solely by the news content of the newly released 
data. ${ }^{14}$ If the new data, i.e., the indicator releases, came in as expected by the model, the news content is zero and the business cycle index will not be revised. Therefore, the new estimate of the index, $E\left[B C I_{t} \mid \Omega_{v+1}\right]$, can be decomposed as proposed in Banbura and Modugno (2014):

$$
\underbrace{E\left[B C I_{t} \mid \Omega_{v+1}\right]}_{\text {New estimate }}=\underbrace{E\left[B C I_{t} \mid \Omega_{v}\right]}_{\text {Old estimate }}+\underbrace{E\left[B C I_{t} \mid I_{v+1}\right]}_{\text {Revision }}
$$

where $\Omega_{v}$ denotes the old data set, $\Omega_{v+1}$ the new data set and $I_{v+1}$ is the news content (newly released data which was unexpected to the model) of the new data set. ${ }^{15}$

To assess the news content of the newly released data, the revision is decomposed further as

$$
E\left[B C I_{t} \mid I_{v+1}\right]=E\left[B C I_{t} I_{v+1}^{\prime}\right] E\left[I_{v+1} I_{v+1}^{\prime}\right]^{-1} I_{v+1}
$$

To calculate $E\left[B C I_{t} I_{v+1}^{\prime}\right]$ and $E\left[I_{v+1} I_{v+1}^{\prime}\right], y_{i_{j}, t_{j}}$, the data release $j$ which corresponds to the observation of indicator $i$ for time period $t$ and decompose $I_{v+1, j}$, is defined as

$$
I_{v+1, j}=y_{i_{j}, t_{j}}-E\left[y_{i_{j}, t_{j}} \mid \Omega_{v}\right]=\Lambda_{i_{j}}\left(f_{t_{j}}-E\left[f_{t_{j}} \mid \Omega_{v}\right]\right)+u_{i_{j}, t_{j}}
$$

by applying our factor model process (2). Therefore,

$$
E\left[B C I_{t} I_{v+1, j}\right]=\Lambda_{g d p} E\left[\left(f_{t_{j}}-E\left[f_{t_{j}} \mid \Omega_{v}\right]\right)\left(f_{t_{j}}-E\left[f_{t_{j}} \mid \Omega_{v}\right]\right)^{\prime}\right] \lambda_{i_{j}}^{\prime} .
$$

Furthermore, the covariance between two data releases $j$ and $l$ is given by

$$
E\left[I_{v+1, j} I_{v+1, l}\right]=\Lambda_{i_{j}} E\left[\left(f_{t_{j}}-E\left[f_{t_{j}} \mid \Omega_{v}\right]\right)\left(f_{t_{l}}-E\left[f_{t_{l}} \mid \Omega_{v}\right]\right)^{\prime}\right] \lambda_{i_{l}}^{\prime}+1_{\{j=l\}} R_{j j} .
$$

The expectation terms on the factors can be obtained from the Kalman smoother. Finding a vector $B_{v+1}$, the revision can be written as a weighted average of the news in

\footnotetext{
${ }^{14}$ Revisions of past data may be due to updated indicator estimates provided by the statistical agencies or due to revisions stemming from the seasonal adjustment procedure.

${ }^{15}$ Note that since the BCI is obtained using the fitted value for GDP, $E\left[B C I_{t} \mid \cdot\right]$ actually equals $E\left[g d p_{t} \mid \cdot\right]$.
} 
Figure 9: News contributions over time for 2015Q1

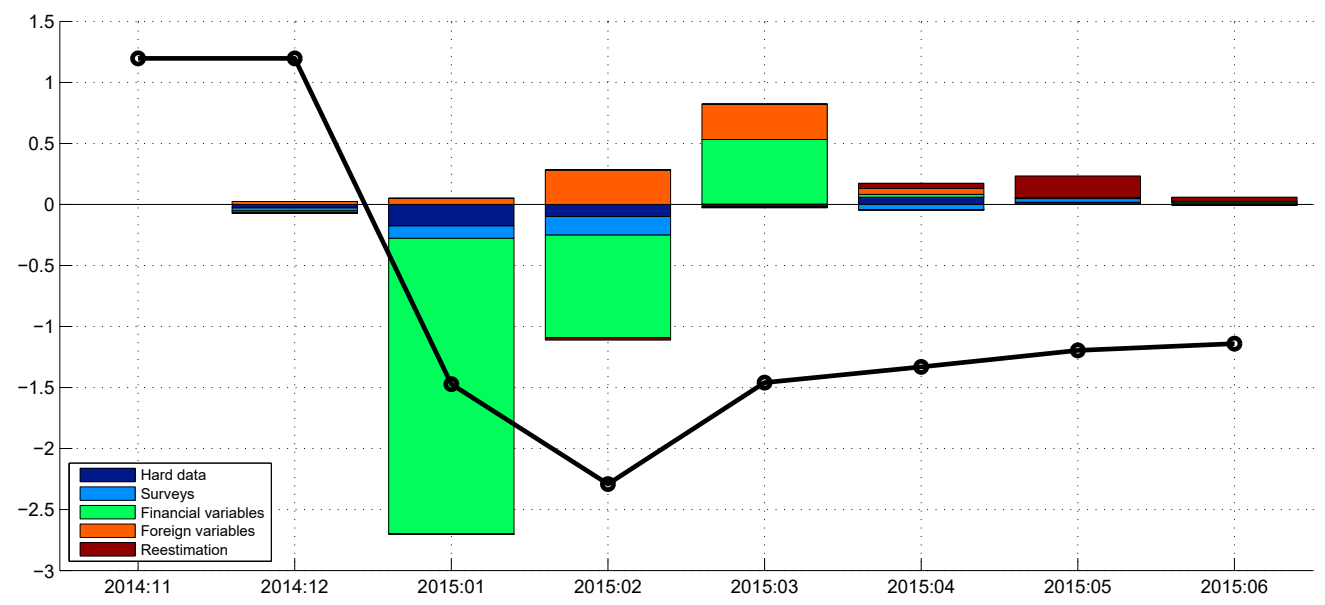

Note: Shown are the revision of the average business cycle index level in 2015Q1 (solid line) and the decomposed contribution of news by indicator type for all indicator releases in the respective month.

the latest release:

$$
E\left[B C I_{t} \mid I_{v+1}\right]=B_{v+1} I_{v+1}=\sum_{j=1}^{J_{v+1}} b_{v+1, j} \underbrace{\left(y_{i_{j}, t_{j}}-E\left[y_{i_{j}, t_{j} \mid \Omega_{v}}\right]\right)}_{\text {News }} .
$$

As an illustration, we investigate the role of news and their contribution to revisions in the business cycle index using an empirical exercise. On January 15, 2015, the Swiss National Bank discontinued the minimum EURCHF exchange rate. This led to an appreciation of the Swiss franc against the euro of approximately $13 \%$ and a substantial slowdown in economic activity in Switzerland. To examine how the different information flows led to revisions in the business cycle index for the quarter of the exchange rate shock, Figure 9 shows the contribution of news to the BCI estimate for 2015Q1 at the end of every month, beginning in November 2014 and ending in July 2015.

The BCI estimate for 2015Q1 started to deteriorate strongly already in January to a level that was compatible with GDP growth of around $-1.5 \%$ (at an annualized rate). This strong downward revision was mainly caused by news from financial indicators. In February, the BCI estimate for 2015Q1 decreased further. The downward revision was again mainly driven by financial variables (for February), mirroring the fact that the exchange rate appreciation was more permanent than the model had initially predicted. To some extent the downward revision was also driven by news from releases of (January) surveys, which decreased even more than the model had anticipated. Foreign indicators, in 
Figure 10: Average contribution of news to BCI revision (percentage share of total)
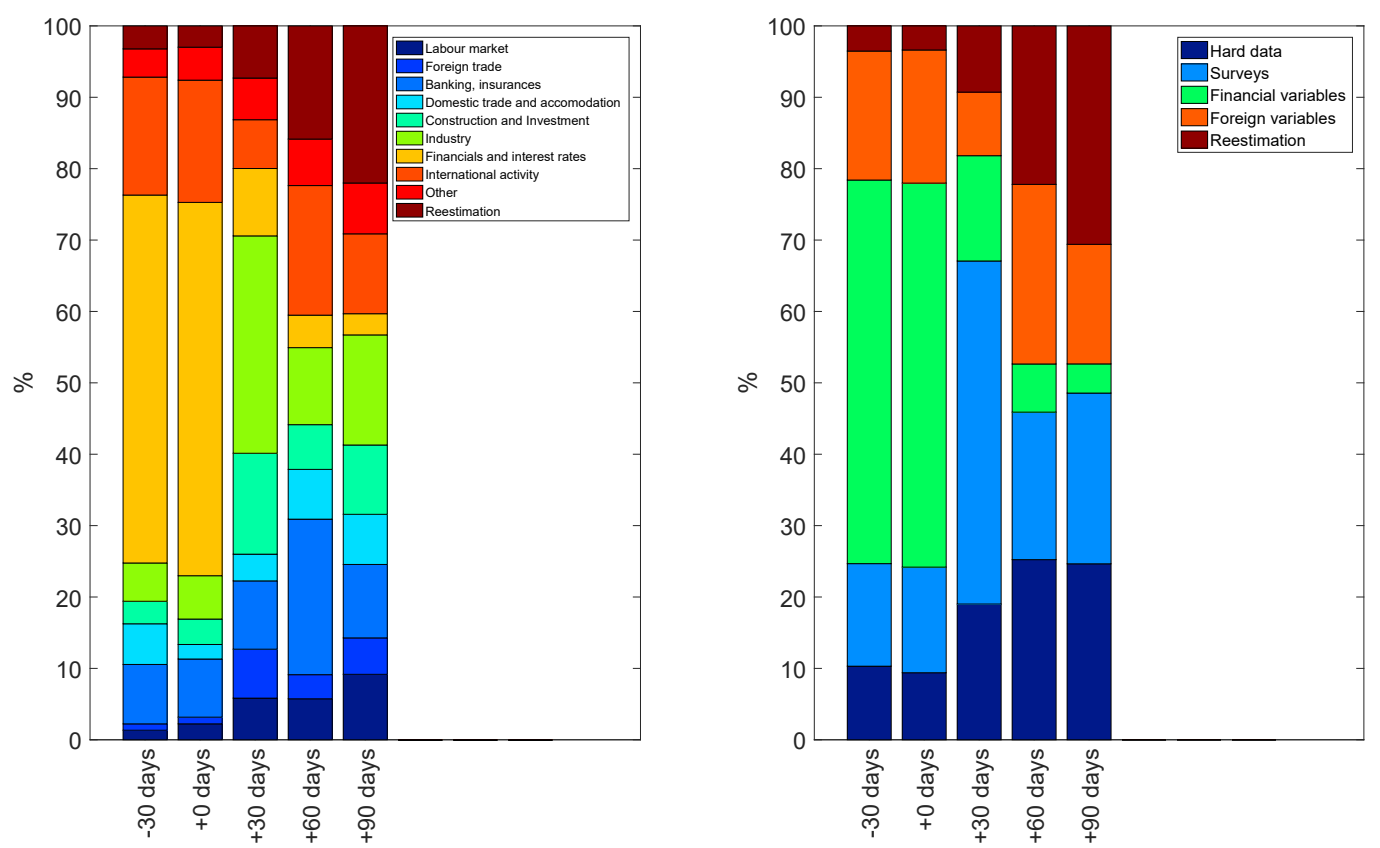

Note: Shown are the average contribution of news (as a percentage share of the total) by indicator group (left panel) and indicator type (right panel) as a function of the number of days to the end of the target month. The average sum of absolute revisions over indicator categories equals 0.85 at the end of the target month, 0.6030 days after, 0.2260 days after and 0.1190 days after. Over indicator types, the average sum of absolute revisions is approximately 0.05 lower for each of the distances to the target month.

contrast, contributed positively. This reflected the fact that the exchange rate shock only affected domestic but not foreign indicators, so that foreign activity was more supportive than the model had predicted.

In March, the BCI estimate for 2015Q1 recovered somewhat. This upward revision was partly due to a slight improvement in some financial variables, namely stock markets dynamics. In addition, the support from foreign variables surprised again to the upside. The news contribution from soft and hard data was zero, which shows that, overall, these indicator releases (mostly for January and February) turned out as (negative as) expected by the model.

From March on, revisions in the BCI estimate for 2015Q1 were limited, indicating that all remaining releases for Q1 (surveys for March and Q1, as well as hard data for February, March and Q1) turned out as expected by the model. In June the business cycle index reached a level that was compatible with GDP growth of around $-1.2 \%$ (annualized).

To investigate the role of news and their contribution to revisions in the business cycle index in a more general way, Figure 10 shows the average contribution share (in 
percent) of news by indicator group and indicator type as a function of the distance to the end of the target month. In the 30 days before the target month and during the target month itself, the majority of the BCI revisions are, on average, driven by news from financial indicators. In contrast, in the 30 days after the target month, the news mainly come from surveys. Later (more than 30 days after the end of the target month), releases consisted of an increasing share of hard data news, but the news contribution from surveys remains important, too.

\subsection{Financial and foreign variables: Information versus noise}

In this section, I analyze in more detail the role of financial variables and foreign indicators that are included in the data set, and in turn enter the business cycle index, in addition to the soft and hard data for the Swiss economy. The potential benefit from the inclusion of financial variables is that they may not only provide a very timely picture of current and expected financial conditions that consumers and firms face but also respond to changes in business and consumer confidence. Moreover, financial developments have a direct impact on turnover in the banking sector. Indicators on foreign economic activity are important for a small open economy such as that of Switzerland in two ways, directly by measuring changes in external demand conditions, and indirectly through their effect on confidence of Swiss export-oriented businesses.

To investigate the impact of financial and foreign variables on the business cycle index, an additional version of the index based only on domestic non-financial indicators, i.e., excluding foreign and financial indicators, is calculated. Figure 11 shows 7 -month moving averages of the resulting business cycle index and compares it with the version that incorporates the entire data set. Overall, the business cycle resulting from the reduced data set including only domestic non-financial variables is very similar to the general data set including all variables. Nevertheless, focusing on the peaks and troughs, there are some noticeable differences.

The effect of the additional information from financial and foreign variables depends on the economic period. For the 1990s, the index based only on domestic non-financial variables is somewhat more cyclical and closer to actual GDP developments. For the 2000s, the opposite is the case: a reduced index would miss the double-dip in $2002 / 3$, point to a 
Figure 11: The impact of financial and foreign variables

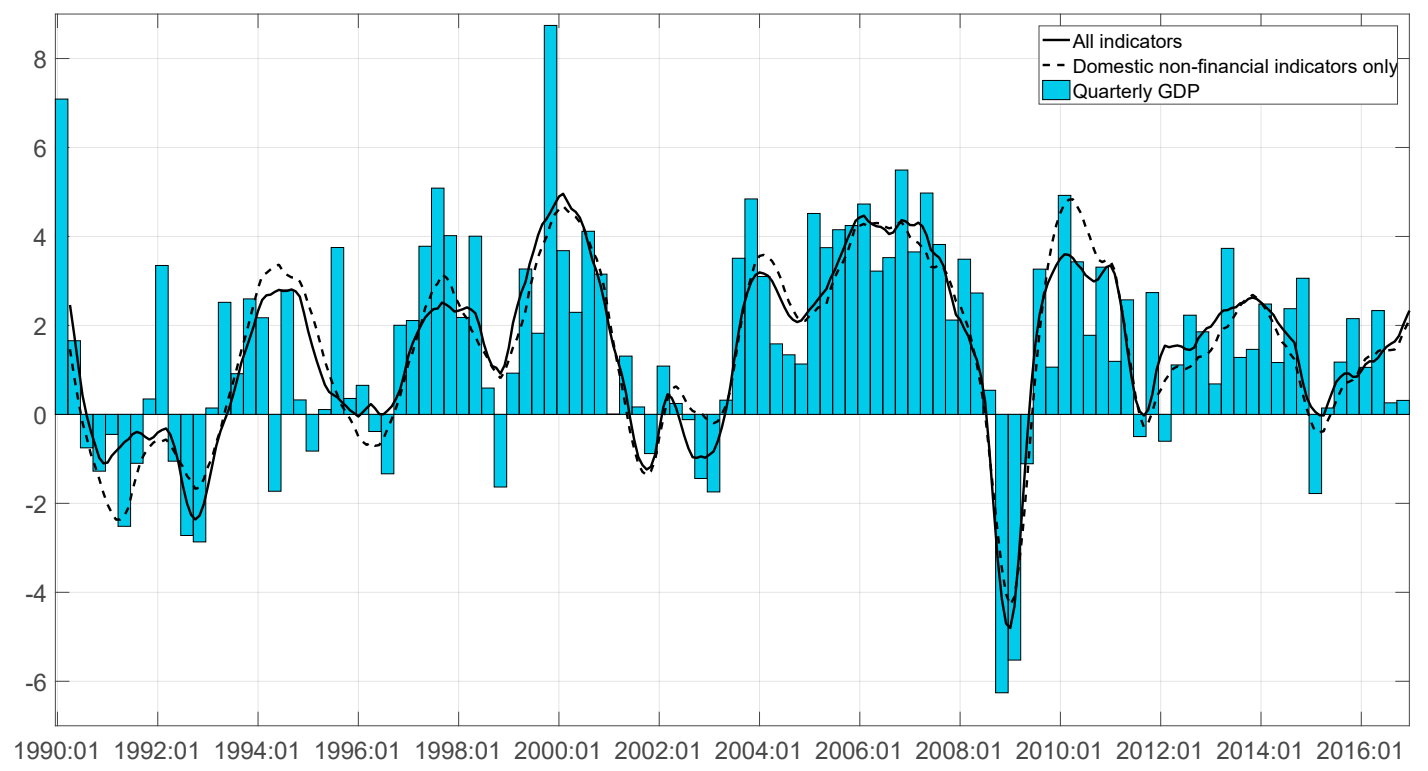

Note: Shown are the 7-month moving averages of the business cycle index based on the entire data set (solid line) and a version based on domestic non-financial variables only (dashed line).

marginally less severe recession in 2008/9 and to a much stronger acceleration after than was actually the case in terms of GDP developments. This may be related to the fact that these crises were caused by financial market turmoil. In contrast, a business cycle index not including financial and foreign variables would see a somewhat more intense downturns following the exchange rate appreciations in 2011 and 2015 and more sluggish recoveries afterwards. This reflects the fact that after these shocks, financial variables, in particular stock market indicators, recovered much more quickly than most domestic non-financial indicators, i.e., hard data, and surveys.

Overall, the correlation between the business cycle index and GDP decreases slightly, by approximately $6 \%$ for both the 1990-2016 sample and the 2000-20016 sample when financial and foreign variables are omitted.

In addition to these in-sample characteristics, there may be other arguments for and against the inclusion of foreign and financial variables, depending on the purpose of the estimated business cycle index, the importance of timeliness or the emphasis on early warning features. The latter can be related to the contribution of news as discussed in the previous section.

To assess how a reduced business cycle index based only on domestic non-financial variables would have reacted to the exchange rate shock in 2015, Figure 12 shows the 
Figure 12: News contributions over time for 2015Q1, domestic non-financial index

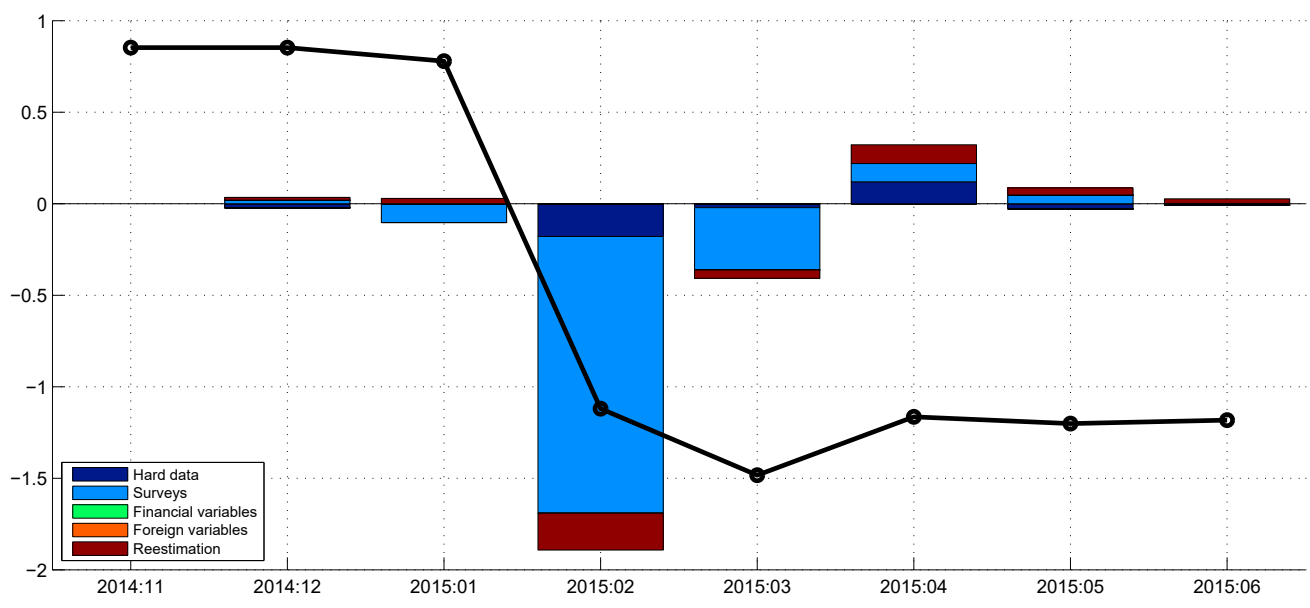

Note: Shown are the revision of the average business cycle index level in 2015Q1 (solid line) and the decomposed contribution of news by indicator type for all indicator releases in the respective month.

related contribution of news. In contrast to the business cycle index using the entire data set, the main news for the reduced index is contained in the surveys. Furthermore, the model reacts one month later, in February, to the changes in the economic environment. The slight undershooting already visible in the general index occurs one month later (in March instead of February), as does the rebound (in April instead of March). However, the reduced BCI estimate for Q1 2015 that is reached in June based on domestic nonfinancial indicators only is very similar to the estimated resulting from the business cycle index using the entire data set.

\subsection{Accuracy within the real-time data flow}

As more and more data become available (see section 4.4), the accuracy of the business cycle index for a given month rises. ${ }^{16}$ This accuracy for a specific point in time (abstracting from parameter uncertainty) can be calculated using the covariance matrix of the smoothed factors, $P_{t \mid T}^{f}$ :

$$
V\left(B C I_{t}\right)=V\left(\Lambda_{g d p} f_{t}\right)=\Lambda_{g d p} \operatorname{Cov}\left(f_{t}\right) \Lambda_{g d p}^{\prime}=\Lambda_{g d p} P_{t \mid T}^{f} \Lambda_{g d p}^{\prime},
$$

\footnotetext{
${ }^{16}$ Note that in this context, the term "accuracy" does not mean closeness to a specific target (since this target, the business cycle, is unobserved in our case). It is more a measure of finality of the index value for a particular month, measuring how much information is already incorporated for this month and the potential for revisions.
} 
Figure 13: Relative accuracy and accuracy gains within the real-time data flow

(a) End-of-month accuracy in terms of distance to target month
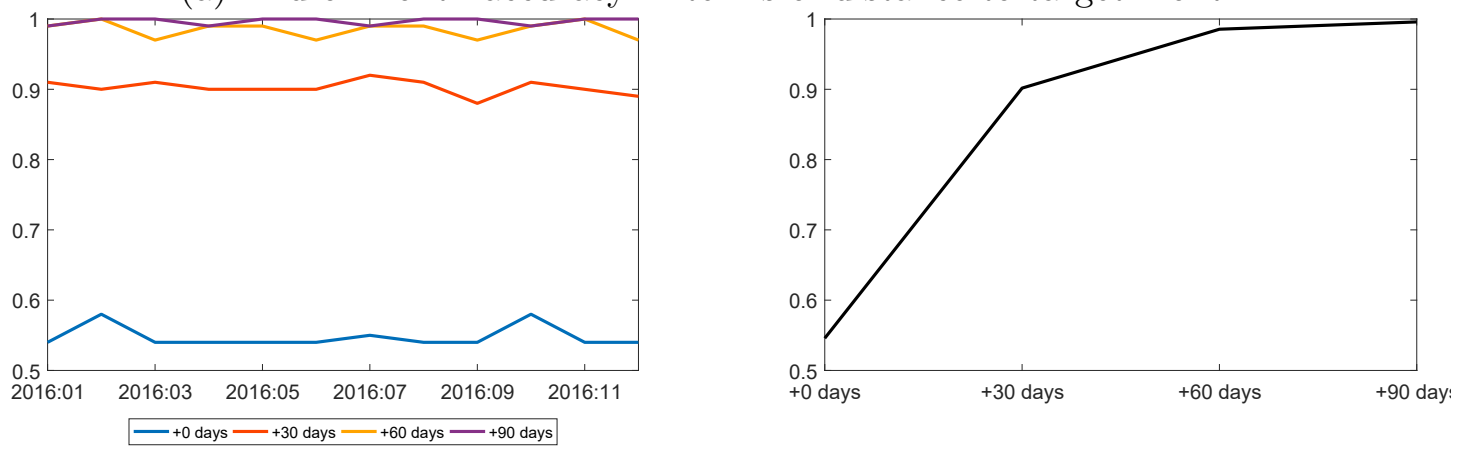

(b) End-of-month accuracy gains in terms of distance to target month, in \%
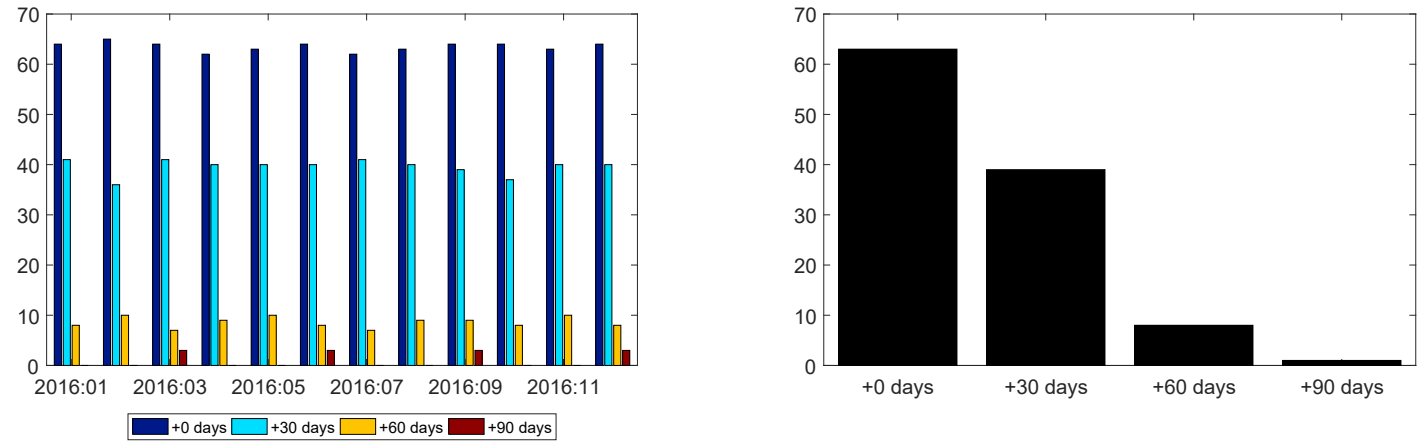

Note: Panel (a) shows the accuracies at the end of the respective month as a function of the distance to the target month (left panel) and the average accuracy as a function of the distance to the target month (right panel). Panel (b) shows the accuracy for the respective month as a function of the distance to the target month (left panel) and the average accuracy gain as a function of the distance to the target month (right panel). Accuracies are calculated relative to the final accuracy when all data have been released. " +30 days", e.g., indicates that the target month has ended 30 days ago.

where $\Lambda_{g d p}$ is the first row of $\Lambda$, which relates to the loadings of GDP on the four BCI factors, and $P_{t \mid T}^{f}$ is the first $4 \times 4$ block of the covariance matrix of the smoothed states, which corresponds to the covariance matrix of the four contemporaneous factors.

Figure 13a plots the accuracy of the BCI at the end of each month (left panel) and on average (right panel) for the example of 2016. Monthly accuracy is calculated relative to the 'final' accuracy when all data for the respective target month have been released. For instance, a value of 0.9 means that accuracy is $10 \%$ lower than the final accuracy will be. The results suggest that, for most months, accuracy is quite high already 30 days after the respective month has ended.

The slight differences in accuracy across months reflect the fact that accuracy gains, shown in Figure 13b, are not the same for each month. There are two reasons for this. First, the release delay of some indicators may vary from month to month. Second, the 
indicators released on a quarterly basis affect the monthly accuracy pattern. This is best visible when looking at the end-of-month accuracies for +60 days. These are usually already close to one, except at the end of March, June, September and December. The reason for this is that for the related +60day-target months (January, April, July and October), information coming from quarterly indicators, in particular quarterly surveys, is not available yet, although 60 days have already passed since the respective target month has ended.

On average, accuracy reaches $55 \%$ of the final accuracy at the end of the respective month, $90 \% 30$ days after and 99\% 60 days after (see right panel of Figure 13a). In terms of accuracy gains (right panel of Figure 13b), accuracy increases on average by $63 \%$ during the target month itself, by an additional $39 \%$ in the next month and by another $8 \%$ the month after.

\section{Conclusions}

For policy institutions such as central banks, it is important to have a timely and accurate measure of past and current economic activity and the business cycle situation. The most prominent example for such a measure is gross domestic product (GDP). However, GDP is only released at a quarterly frequency and with a substantial delay. In Switzerland, for instance, quarterly GDP is published approximately two months after the respective quarter has ended.

In this paper, I constructed a new business cycle measure for the Swiss economy that uses state-of-the-art methods and (a) can be calculated in real-time, even when some indicators are not available yet for the most recent periods (ragged edge setup), (b) is available at a monthly frequency, (c) incorporates a very large number of economic indicators (large-scale setup) and (d) includes both quarterly and monthly indicators (mixedfrequency setup). The two last points are necessary since the index uses a large and broad-based set of monthly and quarterly indicators.

From a detailed assessment of the resulting business cycle index, five findings emerge. First, an analysis of the relative importance of the indicators for the business cycle index suggests that the 100 most important indicators account for only $52 \%$ of the index. This 
is in contrast to the results for other countries. The obtained business cycle index is therefore driven by a large and broad based set of indicators and not only by a small subset of variables. This makes the index much more robust.

Second, the contributions of the different indicators to the index over time shows that although there is a considerable degree of comovement across indicators there can also be periods over which the indicator categories (e.g., industry sector, foreign trade, construction sector, labor market, etc.) and indicator types (e.g., surveys, hard data, financial indicators, foreign indicators) differ more substantially from each other.

Third, an analysis of the contribution of news within the real-time data flow sheds light on how the business cycle index takes into account new data that have been released since the last estimation. This suggests that financial variables are the main source of news to the model within the target month itself, surveys in the month after and hard data two months after.

Fourth, a more detailed investigation of the role of financial and foreign variables shows that, overall, an index based only on domestic non-financial indicators would be very similar to the index including all variables. However, when the focus is only on the peaks and troughs, there are considerable differences. For instance, not including financial and foreign variables would miss the recession in $2002 / 3$ and point to a much stronger acceleration after the financial crisis in 2008 than was actually the case in terms of GDP developments. The reduced business cycle index also sees a somewhat more intense downturn after the exchange rate appreciation in 2011 and 2015 and a more sluggish recovery thereafter than the official GDP figures suggest.

Fifth, analyzing the sensitivity of the new business-cycle index to the real-time data flow reveals that the index becomes quite accurate already 30 days after the respective target month has ended. Accuracy gains are highest in the target month itself and the month after. 


\section{References}

Abberger, K., M. Graff, B. Siliverstovs, and J.-E. Sturm (2014): "The KOF Economic Barometer, Version 2014: A Composite Leading Indicator for the Swiss Business Cycle," KOF Working Paper Series, 353.

Altissimo, F., A. Bassanetti, R. Cristadoro, M. Forni, M. Lippi, L. Reichlin, AND G. Veronese (2001): "Eurocoin: a real time coincident indicator of the euro area business cycle," CEPR Discussion Paper Series, 3108.

Altissimo, F., R. Cristadoro, M. Forni, M. Lippi, and G. Veronese (2010): "New EuroCOIN: tracking economic growth in real time," The Review of Economics and Statistics, 92.

Alvarez, R., M. Camacho, and G. Perez-Quiros (2016): "Aggregate versus disaggregate information in dynamic factor models," International Journal of Forecasting.

Angelini, E., G. Camba-Mendez, D. Giannone, L. Reichlin, and G. Rünstler (2011): "Short-term forecasts of euro area GDP growth," Econometrics Journal, 14(1), $\mathrm{C} 25-\mathrm{C} 44$.

Aruoba, S. B., F. X. Diebold, and S. Scotti (2009): "Real-Time Measurement of Business Conditions," PDF Journal of Business and Economic Statistics, 27.

BAI, J. (2003): "Inferential theory for factor models of large dimensions," Econometrica, 71.

Banbura, M., D. Giannone, M. Modugno, and L. Reichlin (2013): "Now-Casting and the Real-Time Data Flow," in Handbook of Economic Forecasting, ed. by G. Elliot, and A. Timmermann, vol. 2A, chap. 4, pp. 195-237. Elsevier.

Banbura, M., And M. Modugno (2014): "Maximum likelihood estimation of factor models on data sets with arbitrary pattern of missing data," Journal of Applied Econometrics, 29.

Banbura, M., and G. Ruenstler (2011): "A look into the factor model black box: 
Publication lags and the role of hard and soft data in forecasting GDP," International Journal of Forecasting, 27.

Boivin, J., AND S. NG (2006): "Are more data always better for factor analysis?," Journal of Econometrics, 132.

Camacho, M., and G. Perez-Quiros (2010): "Introducing the euro-sting: short-term indicator of euro area growth," Journal of Applied Econometrics, 25.

Crone, T. M., and A. Clayton-Matthews (2005): "Consistent Economic Indexes for the 50 States," Review of Economics and Statistics, 87.

Doz, C., D. Giannone, and L. Reichlin (2011): "A two-step estimator for large approximate dynamic factor models based on Kalman filtering," Journal of Econometrics, 164.

Doz, C., D. Giannone, and L. Reichlin (2012): "A quasi-maximum likelihood approach for large, approximate dynamic factor models," The Review of Economics and Statistics, 94 .

Durbin, J., And S. K. Koopman (2012): Time series analysis by state space models. Oxford University Press.

Forni, M., M. Hallin, M. Lippi, and L. Reichlin (2000): "The genegeneral dynamic factor model: identification and estimation," The Review of Economics and Statistics, 82.

Forni, M., M. Hallin, M. Lippi, and L. Reichlin (2001): "Coincident and leading indicators for the euro area," The Economic Journal, 111.

Frale, C., M. Marcellino, G. Mazzi, and T. Prioetti (2010): "Survey Data as Coincident or Leading Indicators," Journal of Forecasting, 29.

Galli, A., C. Hepenstrick, and R. Scheufele (2017): "Mixed-frequency models for tracking economic developments in Switzerland," SNB Working Paper Series, 2017-2.

Giannone, D., L. Reichlin, and D. Small (2008): "Nowcasting: The real-time informational content of macroeconomic data," Journal of Monetary Economics, 55. 
Hamilton, J. D. (1994): Time Series Analysis. Princeton University Press, Princeton, N.J.

Koopman, S. J., ANd A. HARvey (2003): "Computing observation weights for signal extraction and filtering," Journal of Economic Dynamics \& Control, 27.

Lopes, H. F., And M. West (2004): "Bayesian model assessment in factor analysis," Statistica Sinica, 14.

Luetkepohl, H. (2005): New introduction to multiple time series analysis. SpringerVerlag.

Mariano, R. S., and Y. Murasawa (2003): "A new coincident index of business cycles based on monthly and quarterly series," Journal of Applied Econometrics, 18, 427-443.

Mariano, R. S., and Y. Murasawa (2010): "A coincident index, common factors, and monthly real gdp," Oxford Bulletin of Economics and Statistics, 72.

Matheson, T. (2011): "New indicators for tracking growth in real time," IMF Working Paper, $11 / 43$.

Matheson, T. D. (2012): "Financial conditions indexes for the United States and euro area," Economics Letters, 115, 441-446.

Neusser, K. (2011): Zeitreihenanalyse in den Wirtschaftswissenschaften. Vieweg + Teubner, Wiesbaden, 3rd edn.

Siliverstovs, B. (2013): "Dating business cycles in historical perspective: Evidence for Switzerland," Journal of Economics and Statistics (Jahrbuecher fuer Nationaloekonomie und Statistik), 233.

Song, J., AND T. R. BeLIN (2008): "Choosing an appropriate number of factors in factor analysis with incomplete data," Computational Statistics and Data Analysis, 52.

Stock, J. H., And M. W. Watson (2002a): "Forecasting using principal components from a large number of predictors," Journal of the American Statistical Association, 97.

(2002b): "Macroeconomic forecasting using diffusion indexes," Journal of Business $\&$ Economic Statistics, 20. 
(2011): "Dynamic factor models," in The Oxford Handbook of Economic Forecasting, ed. by M. P. Clements, and D. F. Hendry, chap. 7, pp. 35-59. Oxford University Press.

The Conference Board (2001): Business Cycle Indicators Handbook. 


\section{A The Kalman filter}

Following Hamilton (1994), for our state space model

$$
\begin{gathered}
y_{t}=H_{t} \xi_{t}+\epsilon_{t} \\
\xi_{t}=F \xi_{t-1}+e_{t} \\
\text { with }\left[\begin{array}{l}
e_{t} \\
\epsilon_{t}
\end{array}\right] \sim N\left[\begin{array}{ll}
Q & 0 \\
0 & R
\end{array}\right],
\end{gathered}
$$

the Kalman filter applied with the parameters $\theta=\left[\begin{array}{llll}\Lambda^{*} & \Phi & \Sigma_{v v} & \Sigma_{w w}\end{array}\right]^{\prime}$ provides a time series of filtered states $\left\{\xi_{i \mid i}\right\}_{i=1}^{T}$ and their covariance matrices $\left\{P_{i \mid i}\right\}_{i=1}^{T}$. The Kalman smoother provides a time series of the smooth correspondences, $\left\{\xi_{i \mid T}\right\}_{i=1}^{T}$ and $\left\{P_{i \mid T}\right\}_{i=1}^{T}$.

\section{Details on the Kalman procedure}

First we define:

- $\xi_{t \mid t-1}=E\left[\xi_{t} \mid\left\{y_{i}\right\}_{i=1}^{t-1}\right]$, the forecast for $\xi_{t}$ given data on $y$ up to t-1

- $y_{t \mid t-1}=E\left[y_{t} \mid\left\{y_{i}\right\}_{i=1}^{t-1}\right]$, the forecast for $y_{t}$ given data on $y$ up to t-1

- $P_{t \mid t-1}=\operatorname{Var}\left(\xi_{t} \mid\left\{y_{i}\right\}_{i=1}^{t-1}\right)$, the covariance matrix of $\xi_{t}$

- $\xi_{t \mid t}=E\left[\xi_{t} \mid\left\{y_{i}\right\}_{i=1}^{t}\right]$, the update for $\xi_{t}$ given all information up to $\mathrm{t}$

- $\left.P_{t \mid t}=\operatorname{Var}\left(\xi_{t} \mid y_{i}\right\}_{i=1}^{t}\right)$, the covariance of $\xi_{t}$ given all information up to $\mathrm{t}$

Given our parameters $\theta$, the Kalman filter is then just an application of theorems on two variables (in our case $\xi$ and $y$ ) that are jointly normal distributed. The estimation proceeds as follows:

- Step 1, forecasting $\xi_{t}: \xi_{t \mid t-1}=F \xi_{t-1 \mid t-1}$

- Step 2, variance of forecast for $\xi_{t}: P_{t \mid t-1}=F P_{t-1 \mid t-1} F^{\prime}+Q$

- Step 3, forecasting $y_{t}: y_{t \mid t-1}=H_{t} \xi_{t \mid t-1}$ 
- Step 4, variance of forecast for $y_{t}: S_{t}=H_{t} P_{t \mid t-1} H_{t}^{\prime}+R$

- Step 5, forecast error of $y_{t}: z_{t}=y_{t}-y_{t \mid t-1}$

- Step 6, updating $\xi_{t}: \xi_{t \mid t}=\xi_{t \mid t-1}+P_{t \mid t-1} H_{t}^{\prime} S_{t}^{-1} z_{t}$

- Step 7, variance of update for $\xi_{t}: P_{t \mid t}=P_{t \mid t-1}-P_{t \mid t-1} H_{t}^{\prime} S_{t}^{-1} H_{t} P_{t \mid t-1}$

- Step 8, Kalman gain: $K_{t}=F P_{t \mid t-1} H_{t}^{\prime} S_{t}^{-1}$

- Do the same for $\left\{\xi_{t+1}, P_{t+1}\right\},\left\{\xi_{t+2}, P_{t+2}\right\}$, and so on.

The result is a time series of filtered states $\left\{\xi_{i \mid i}\right\}_{i=1}^{T}$ and their covariance matrices $\left\{P_{i \mid i}\right\}_{i=1}^{T}$. In the filtered state vector, missing values have been filled by the Kalman filter.

\section{Details on the smoothing procedure}

From the Kalman filter, we obtain estimates of $\xi_{t \mid t-1}$ and $P_{t \mid t-1}$ (the forecasts, based on past information) as well as of $\xi_{t \mid t}$ and $P_{t \mid t}$ (the updates, based on past and current information) for each time period 1 to T. Starting with $\xi_{T \mid T}$ (the update for the last time period), we can go backwards to smooth our estimates by also incorporating future estimates and information, i.e., the estimation error for the next period given all information.

For $t=T$, the smoothed estimate is simply given by the filtered state, i.e.

$$
\xi_{t \mid T}=\xi_{t \mid t}
$$

and for $t<T$ by

$$
\xi_{t \mid T}=\xi_{t \mid t}+P_{t \mid t} F^{\prime} P_{t+1 \mid t}^{-1}\left(\xi_{t+1 \mid T}-\xi_{t+1 \mid t}\right)
$$

The result is a time series of smoothed states $\left\{\xi_{i \mid T}\right\}_{i=1}^{T}$. The mean squared error (MSE) of the smoothed states $\xi_{t \mid T}$ is given by

$$
P_{t \mid T}=P_{t \mid t}+J_{t}\left(P_{t+1 \mid T}-P_{t+1 \mid t}\right) J_{t}^{\prime}
$$




\section{B Additional figures and tables}

Figure B.1: The four factors of the business-cycle index
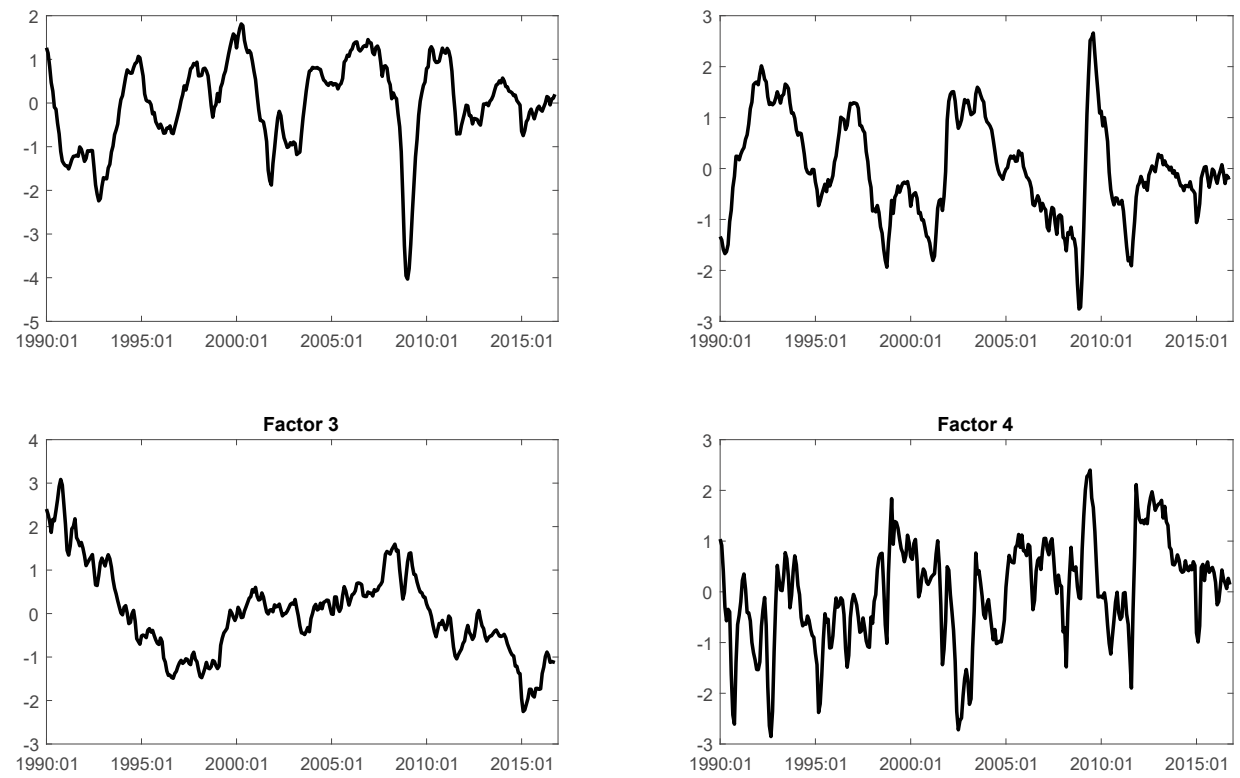

Note: Shown are the four BCI factors.

Figure B.2: Separate contributions to business cycle index by indicator categories
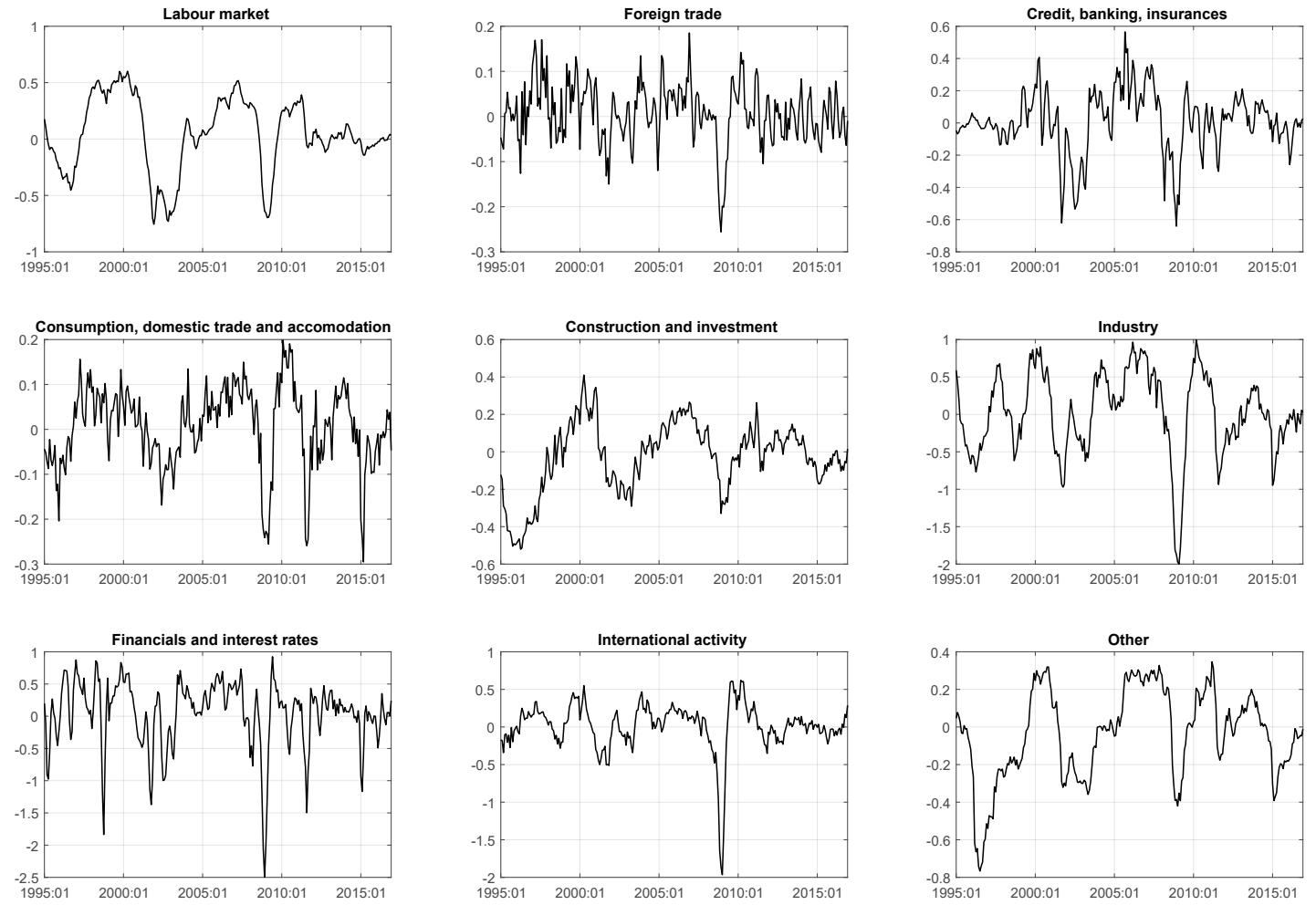

Note: Shown are the BCI contributions from the different indicator categories in deviations from mean. 
Figure B.3: Contributions to business cycle index by indicator types

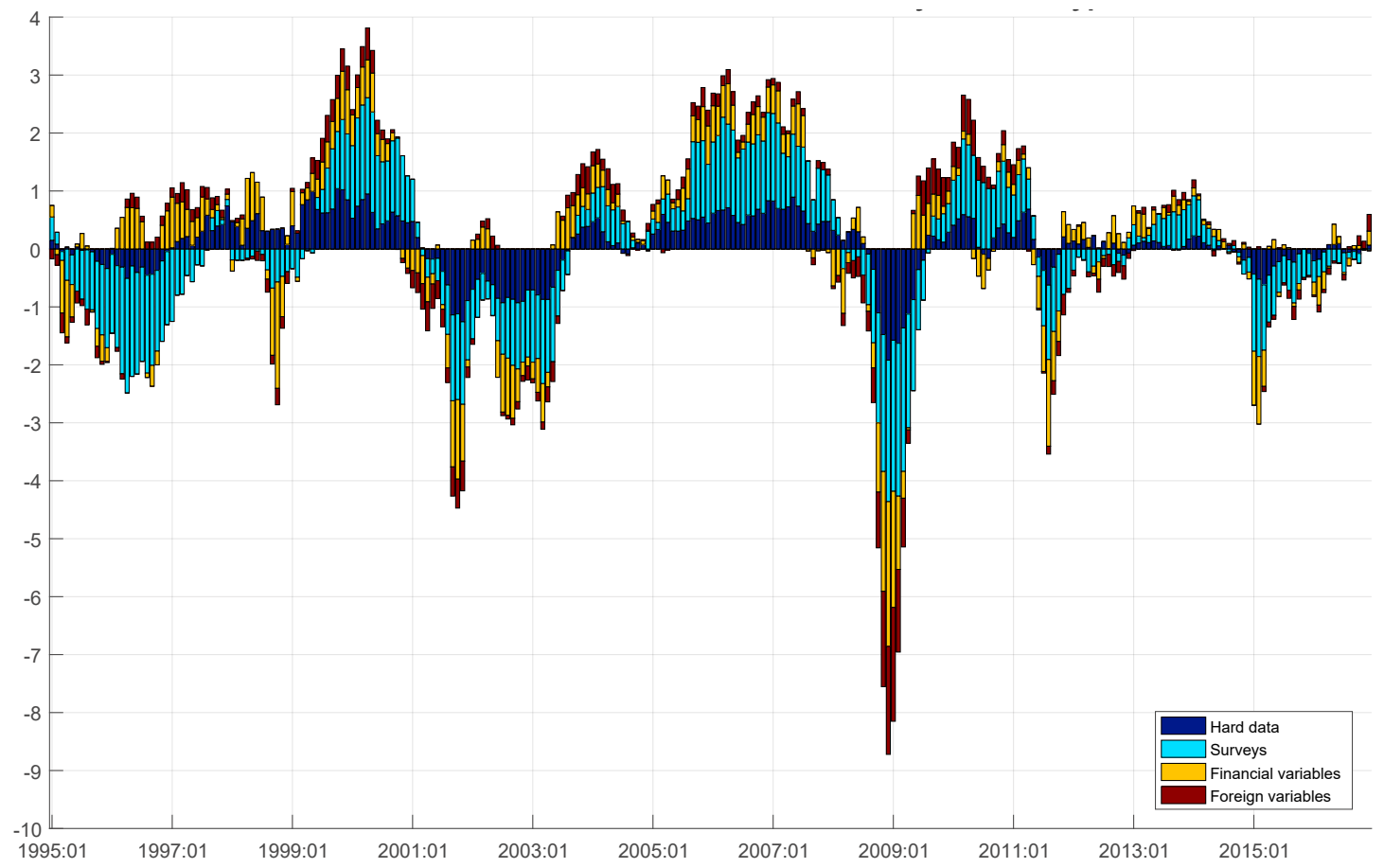

Note: Shown are the BCI contributions from the different indicator types in deviations from mean.

Figure B.4: Separate contributions to business cycle index by indicator types
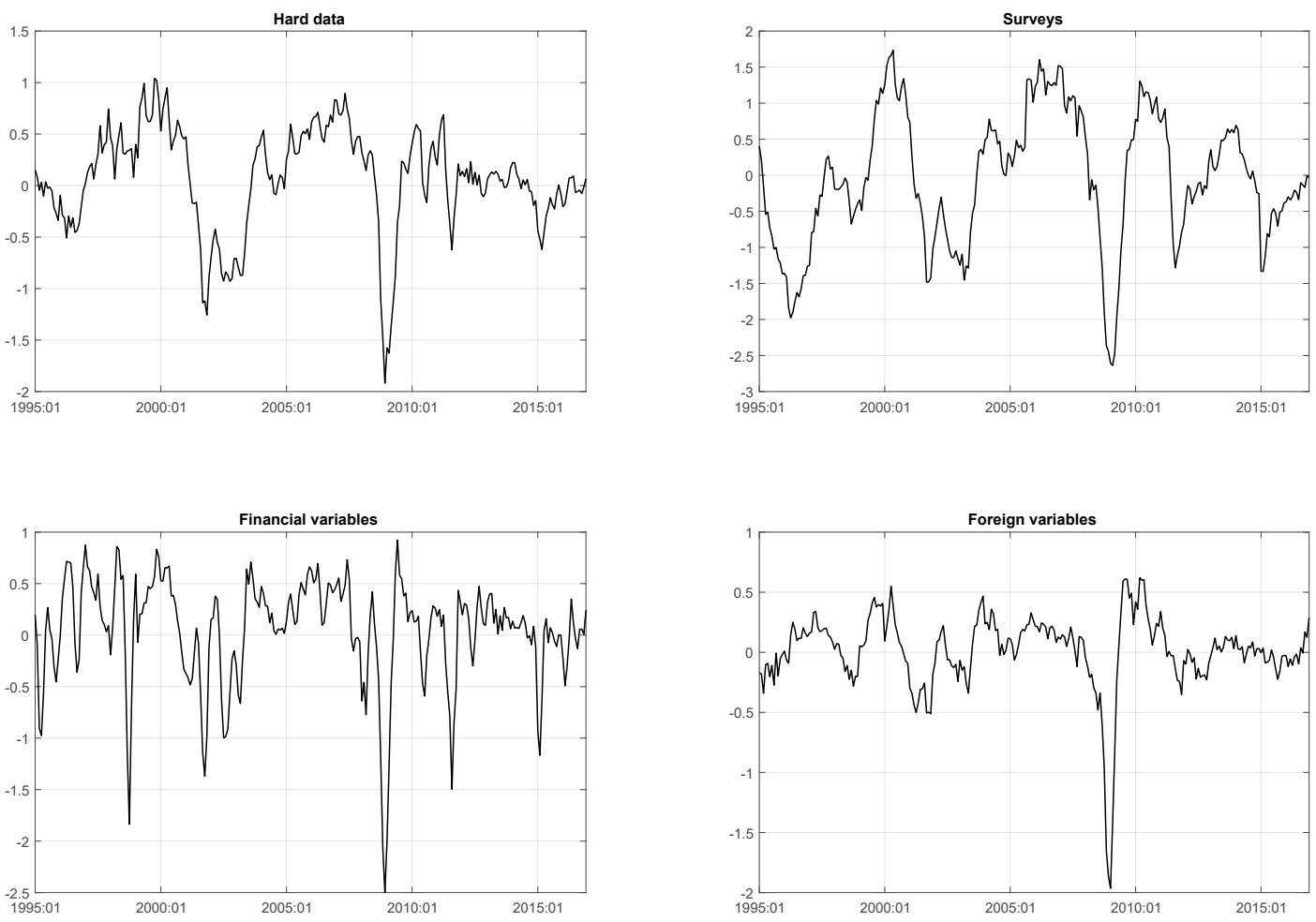

Note: Shown are the BCI contributions from the different indicator types in deviations from mean. 
Table B.1: Legend for the acronyms of the 30 most important indicators

\begin{tabular}{|c|c|}
\hline Acronym & Indicator \\
\hline INDKOFTOTBESCHE & KOF industry survey: employment expectations \\
\hline BANSECHOLDING & Security holdings of banks \\
\hline BAUKOFPREIE & KOF construction survey on selling price expectations \\
\hline FINSPI & Swiss performance index \\
\hline FINOECDUBS100 & UBS100 index-fund \\
\hline BANWERTSCHR & Bank deposits of domestic customers \\
\hline INDKOFTOTPLAN & KOF industry survey: business plans \\
\hline INDKOFTOTVORPRODEINKE & KOF industry survey: expected purchases of intermediate goods \\
\hline FINSMI & Swiss market index \\
\hline INTWLDPMIMANU & World manufacturing PMI \\
\hline FINEXRNEER24 & Nominal export-weighted effective exchange rate (24 countries) \\
\hline INDKOFTOTPRODE & KOF industry survey: expected production \\
\hline INTEWPMI & Export-weighted foreign manufacturing PMIs \\
\hline INDKOFTOTBESTEINGE & KOF industry survey: expected new orders \\
\hline FINGOVBOND10Y & 10-year government bond rate \\
\hline FINEXRCHGBP & Nominal exchange rate of CHF against GBP \\
\hline FINEXRREER24 & Real export-weighted effective exchange rate (24 countries) \\
\hline FINEXRGBPR & Real exchange rate of $\mathrm{CHF}$ against GBP \\
\hline INDKOFTOTAUFTRAGVM & KOF industry survey: backlog of orders \\
\hline FINLIBOR12 & 12-month LIBOR \\
\hline BAUKOFNACHFRE & KOF construction survey: expected demand \\
\hline AIUKOFGESCHE & KOF project engineering survey: expected business conditions \\
\hline LABUNEMPFUNCSPEC & Number of unemployed by function: Skilled workers \\
\hline FINHYPO2Y & 2-year mortgage rate \\
\hline AIUKOFDEME & KOF project engineering survey: expected demand \\
\hline LABUNEMPDHL & Unemployment by sector: retail trade \\
\hline FINMSCIWLD & MSCI world index \\
\hline INTDEORDHALBF & German industry orders of intermediate goods \\
\hline INDKOF67BESCHE & $\begin{array}{l}\text { KOF industry survey: expected employment (export-oriented firms } \\
\text { only) }\end{array}$ \\
\hline FINEXRCHEUR & Nominal exchange rate, CHF against the EUR \\
\hline
\end{tabular}


Figure B.5: Availability of indicators in the data set


Note: Shown is the availability of all quarterly (top panel) and monthly (bottom panel) indicators in the data set. Green colors indicate that the indicator is available for the respective period, red colors indicate that the indicator is unavailable. 


\section{Recent SNB Working Papers}

2017-8 Alain Galli: Which indicators matter? Analyzing the Swiss business cycle using a large-scale mixedfrequency dynamic factor model.

2017-7 Gregor Bäurle, Matthias Gubler and Diego R. Känzig: International inflation spillovers - the role of different shocks.

2017-6 Lucas Marc Fuhrer: Liquidity in the Repo Market.

2017-5 Christian Grisse, Signe Krogstrup and Silvio Schumacher: Lower bound beliefs and long-term interest rates.

2017-4 Toni Beutler, Robert Bichsel, Adrian Bruhin and Jayson Danton: The Impact of Interest Rate Risk on Bank Lending.

2017-3 Raphael A. Auer, Andrei A. Levchenko and Philip Sauré: International Inflation Spillovers Through Input Linkages.

2017-2 Alain Galli, Christian Hepenstrick and Rolf Scheufele: Mixed-frequency models for tracking short-term economic developments in Switzerland.

2017-1 Matthias Gubler and Christoph Sax: The Balassa-Samuelson Effect Reversed: New Evidence from OECD Countries.

2016-19 Jens H.E. Christensen and Signe Krogstrup: A Portfolio Model of Quantitative Easing.

2016-18 Jonas Meuli, Thomas Nellen and Thomas Nitschka: Securitisation, loan growth and bank funding: the Swiss experience since 1932 .
2016-17 Rita Fleer, Barbara Rudolf and Mathias Zurlinden: Price change dispersion and time-varying pass-through to consumer prices.

2016-16 Gregor Bäurle, Daniel Kaufmann, Sylvia Kaufmann and Rodney W. Strachan: Changing dynamics at the zero lower bound.

2016-15 Adrian Jäggi, Martin Schlegel and Attilio Zanetti: Macroeconomic surprises, market environment and safe-haven currencies.

2016-14 Alain Galli: Sticky consumption and wealth effects in Switzerland.

2016-13 Gregor Bäurle and Rolf Scheufele: Credit cycles and real activity - the Swiss case.

2016-12 Silvio Schumacher: Networks and lending conditions: Empirical evidence from the Swiss franc money markets.

2016-11 Lucas Marc Fuhrer, Benjamin Müller and Luzian Steiner: The Liquidity Coverage Ratio and Security Prices.

2016-10 Nikola Mirkov, Igor Pozdeev and Paul Söderlind: Toward Removal of the Swiss Franc Cap: Market Expectations and Verbal Interventions.

2016-9 Severin Bernhard and Till Ebner: Cross-border Spill over Effects of Unconventional Monetary Policieson Swiss Asset Prices.

2016-8 Pinar Yeşin: Capital Flows and the Swiss Franc. 
